TOPICAL REVIEW

\title{
Quantum Simulations with Cold Trapped Ions
}

\author{
Michael Johanning, Andrés F. Varón, Christof Wunderlich \\ Fachbereich Physik, Universität Siegen, 57068 Siegen, Germany \\ E-mail: wunderlich@physik.uni-siegen.de
}

Submitted to: J. Phys. B: At. Mol. Opt. Phys.

\begin{abstract}
The control of internal and motional quantum degrees of freedom of laser cooled trapped ions has been subject to intense theoretical and experimental research for about three decades. In the realm of quantum information science the ability to deterministically prepare and measure quantum states of trapped ions is unprecedented. This expertise may be employed to investigate physical models conceived to describe systems that are not directly accessible for experimental investigations. Here, we give an overview of current theoretical proposals and experiments for such quantum simulations with trapped ions. This includes various spin models (e.g., the quantum transverse Ising model, or a neural network), the Bose-Hubbard Hamiltonian, the Frenkel-Kontorova model, and quantum fields and relativistic effects.
\end{abstract}

PACS numbers: 03.67.Ac, 37.10.Ty, 37.10.Vz 


\section{Introduction}

Atomic ions confined in an electrodynamic trap provide us with individual quantum systems whose internal and external degrees of freedom can be controlled to a considerable degree by the experimenter. A strong motivation for early experiments with individual trapped ions arose from their potential use as frequency standards (e.g., [Dehmelt 1981] and references therein). Today, by using trapped ions as frequency standards unsurpassed accuracy and precision has been reached (e.g., [Rosenband et al. 2008] and references therein), and in addition, trapped ions are used for a wide range of investigations into fundamental questions of physics, for instance in the field of quantum information science. This special issue gives an account of the many facets of research with trapped ions.

Controlling motional and internal states of trapped ions by letting them interact with electromagnetic fields, ranging from uv light to rf radiation, has lead to the realization of experiments that previously (reaching well into the second half of the 20th century) were only conceivable, by most physicists, as Gedankenexperiments. A small (and unsystematic) sample from such fascinating experiments include the trapping and visualization of a single ion [Neuhauser et al. 1980], the laser cooling of a single ion to its motional ground state [Diedrich et al. 1989], the deterministic generation and analysis of Schrödinger cat and other entangled states of massive particles, [Monroe, Meekhof, King \& Wineland 1996, Leibfried et al. 2005, Häffner et al. 2005, Blatt \& Wineland 2008], or the teleportation of ions [Riebe et al. 2004, Barrett et al. 2004].

While building a universal quantum computer still poses formidable experimental challenges, in particular because of the low error thresholds required for fault-tolerant computing it appears that useful quantum simulations are more amenable to experimental efforts.

There are two common usages of the term "quantum simulation": (i) the simulation of static and dynamic properties of quantum systems (e.g. atoms, molecules, condensed matter systems) using classical computers, and (ii) the simulation of the properties of one quantum system by means of controlling and observing another quantum system. Here, we are concerned with the second type of research.

The purpose of a quantum simulation is to use a well-understood physical A system to simulate the static properties and the dynamics of another system B that is difficult or, for all practical purposes, even impossible to investigate experimentally [Feynman 1982, Feynman 1986, Jane et al. 2003]. In order to be able to simulate B by observing A, both physical systems need to be described by the same underlying mathematical model.

In this article we give an overview of concrete proposals for performing experimental quantum simulations with laser cooled trapped ions, and of experimental work immediately relevant for using trapped ions as a quantum simulator.

\section{Spin models}

Interacting spins play an important role in many physical models in a variety of research fields of physics, for instance, in models that are employed to describe phenomena in condensed matter physics (e.g., [Schollwöck et al. 2004] and references therein). Also, in recent years the connection between entanglement on the one hand, and static and dynamic properties of many-body systems on the other hand, for instance, near quantum phase transitions has attracted much interest (e.g., [Osterloh et al. 2002, Amico et al. 2008, Plenio \& Virmani 2007] and references therein). 
Probably the simplest approach to modeling interacting spins is the classical Ising model [Lenz 1920, Ising 1925] where the energy of a system of localized particles is given by

$$
E=-J \sum_{\langle i>j\rangle} s_{i} s_{j}
$$

and each particle (spin) maybe in two possible states indicated by $s_{i}= \pm 1$. The summation includes the interaction between nearest neighbours only, indicated by $\langle i>j\rangle$. Beyond interacting spins, the Ising model is often used to approximate systems where the constituents may be in one of two possible states, describing, for example, the tunneling between two potential wells or the occupation of a lattice site with two types of particles [Brush 1967]. Ising already showed that a phase transition between an ordered state and a disordered state does not take place in this model in one dimension at non-zero temperature, whereas in two dimensions such a phase transition is possible as shown by Onsager [Onsager 1944]. However, extending the range of interaction beyond nearest neighbours gives rise to phase transitions even in one dimension [Gitterman \& Halpern 2004].

A more sophisticated model of the interaction between localized spins [Heisenberg 1928] that, in addition, are exposed to an external local magnetic field $\vec{B}_{i}$ uses the Hamiltonian

$$
H_{s}=-\frac{2}{\hbar} \sum_{i>j} J_{i j} \vec{S}_{i} \cdot \vec{S}_{j}-\frac{g \mu_{B}}{\hbar} \sum_{i} \vec{S}_{i} \cdot \vec{B}_{i} .
$$

Here we assume that all lattice sites are populated by particles with spin $\vec{S}_{i}$ with identical $g$-factor and eigenvalues of $S_{z}$ that are multiples of $\hbar / 2\left(\mu_{B}\right.$ is the Bohr magneton). No assumption has yet been made on the physical nature and range of the spin-spin coupling which often is caused by a dipole-dipole or exchange interaction. Therefore, it is useful to explicitly parametrize a possible anisotropy of this coupling by introducing the constants $J_{i j}^{(\alpha)}$, with $\alpha=x, y, z$ so that

$$
H_{s}^{(a)}=-\frac{2}{\hbar} \sum_{\alpha=x, y, z} \sum_{i>j} J_{i j}^{(\alpha)} S_{i}^{(\alpha)} S_{j}^{(\alpha)}-\sum_{i} \vec{S}_{i} \cdot \vec{B}_{i}^{\prime},
$$

where the scaled local fields are defined as $\vec{B}_{i}^{\prime} \equiv \frac{g \mu_{B}}{\hbar} \vec{B}_{i}$.

When describing physical phenomena that occur in condensed matter, for example, concerning magnetism, this Hamiltonian is often subject to idealizations with regard to its dimensionality, the range of interaction, its (an-)isotropy, the uniformity of coupling constants $J_{i j}$, the magnitude and direction of additional (local or global) fields, and regarding the finite size of a particular system under consideration.

The Hamiltonian of the quantum transverse Ising model is obtained from $H_{s}^{(a)}$ for spin$1 / 2$ particles (i.e., $\vec{S}=(\hbar / 2) \vec{\sigma}$ ) by setting $J_{i j}^{(x)}=0=J_{i j}^{(y)}$ and then including further restrictions: Specifically, the local fields $\vec{B}_{i}$ are replaced by a global field $\vec{B}=(B, 0,0)^{T}$ pointing in the $x$-direction, thus neglecting inhomogeneities in the physical system to be modeled [Igloi et al. 1993, Platini et al. 2007]. Additional approximations often made are that the interaction between spins is restricted to nearest neighbours, and uniform coupling $J_{i j}^{(z)}=J \forall i, j$ is assumed. This then leads to

$$
H_{T I}=-\frac{\hbar}{2} J \sum_{\langle i>j\rangle} \sigma_{i}^{z} \sigma_{j}^{z}-B_{x}^{\prime} \sum_{i} \sigma_{i}^{x} .
$$

The quantum transverse Ising model is a prominent example for a spin model [Pfeuty 1970] that has been the starting point for many theoretical investigations (e.g., [Das \& Chakrabarti 2005] Part I and references therein). 


\subsection{Simulation of spin models with trapped ions}

Using two internal states of a trapped ion as an effective spin-1/2 with the ions interacting via the Coulomb force allows for engineering spin couplings by letting the ions interact, in addition, with external dynamic and static fields. Initial theoretical and experimental work was mainly concerned with creating effective interactions between two spins to be used as 2-qubit quantum gates [Cirac \& Zoller 1995, Monroe, Meekhof, King, Itano \& Wineland 1995, Molmer \& Sorensen 1999, Milburn 1999, Milburn et al. 2000, Sørensen \& Mølmer 2000, Jonathan et al. 2000, Jonathan \& Plenio 2001, Cirac \& Zoller 2000, Sasura \& Steane 2003, Schmidt-Kaler et al. 2003, Leibfried et al. 2003]

2.1.1. Direct spin-spin interaction In this section we concentrate on couplings of the type given in equation 3 that, for example, allow to study a phase transition between ordered and disordered phases of an ion crystal as will be outlined in section 2.2 The question we want to consider first is, How can such a spin-spin coupling be obtained in an ion trap and how can it be engineered to simulate a specific Hamiltonian?

If we consider a laser cooled Coulomb crystal of singly charged ions confined in a linear trap such that they arrange themselves in a 1-d string, then the relevant length scale for the inter-ion distance is

$$
\zeta \equiv\left(e^{2} / 4 \pi \epsilon_{0} m \nu_{1}^{2}\right)^{1 / 3},
$$

where $m$ is the mass of one singly charged ion, $e$ the elementary charge, and $\nu_{1}$ is the angular vibrational frequency of the center-of-mass axial mode of the collection of ions [Steane 1997, James 1998]. We have $\zeta=1.5 \times 10^{-5}\left(M f^{2}\right)^{-1 / 3} \mathrm{~m}$ with $M$ in atomic mass units and $f=\nu_{1} / 2 \pi$ in units of MHz. Thus, $\zeta$ typically amounts to a few micrometers. For a linear ion string the inter-ion spacing is $\delta z \approx \zeta 2 N^{-0.56}$ [Schiffer 1993, Dubin 1993] which gives $3.7 \mu \mathrm{m}$ for ${ }^{171} \mathrm{Yb}^{+}$and $9.9 \mu \mathrm{m}$ for ${ }^{9} \mathrm{Be}^{+}$assuming two ions confined in a potential characterized by $f=1 \mathrm{MHz}$.

In principle, a direct spin-spin interaction may take place via a magnetic moment associated with the (pseudo-)spin of the ions or via an exchange interaction. However, as will be recapitulated now, the inter-ion spacing renders this interaction negligible. It is nevertheless instructive to have a look at the dipole-dipole interaction, since it may help to gain an intuitive understanding also of the indirect spin-spin coupling treated in section 2.1.2

For the moment, for the sake of obtaining an intuitive picture, we consider two spins with their relative alignment fixed, and exposed to local magnetic fields $B_{1,2}^{(z)}$ such that the uncoupled Hamiltonian reads $H_{0}=(\hbar / 2)\left(\omega_{1} \sigma_{1}^{(z)}+\omega_{2} \sigma_{2}^{(z)}\right)\left(\omega_{1,2}\right.$ are the angular Larmor precession frequencies). Treating the magnetic dipole-dipole interaction as a perturbation to first order, its magnitude is determined by the expectation value

$$
\left\langle s_{1}, s_{2}\left|V_{M D}\right| s_{1}, s_{2}\right\rangle=s_{1} s_{2} f_{\theta}(\delta z) \equiv \pm \hbar \Delta \omega
$$

where $s_{1,2}= \pm 1$ and

$$
\begin{aligned}
V_{M D} & =-\frac{\hbar^{2}}{4} \frac{\mu_{0}}{4 \pi} \frac{\gamma_{1} \gamma_{2}}{\delta z^{3}}\left(3 \cos ^{2}(\theta)-1\right) \sigma_{1}^{(z)} \sigma_{2}^{(z)} \\
& \equiv f_{\theta}(\delta z) \sigma_{1}^{(z)} \sigma_{2}^{(z)} .
\end{aligned}
$$

Here, the gyromagnetic ratio of spins 1 and 2 is denoted by $\gamma_{1,2}, \mu_{0}$ indicates the permeability of empty space, and $\theta$ is the angle indicating the relative orientation of the two spins. Equation 6 tells us that the energy of the two-spin systems depends on the relative orientation between the two spins, that is, flipping either one of the two spins results in a change of energy. The 
physical reason is that the energy of a given spin depends not only on the imposed local field $B_{1,2}^{(z)}$, but also on the field generated by the second spin at the location of the first one, which in turn depends on its orientation (compare figure 1 and figure 2).

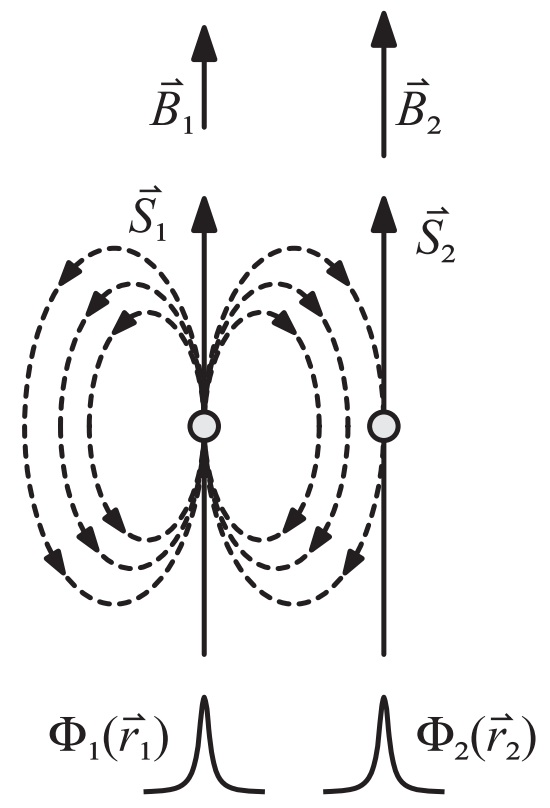

Figure 1. An artist's illustration of the coupling between two spins via a dipole-dipole interaction. Two classical magnetic moments $\gamma_{i} \vec{S}_{i}$ fixed in space are exposed to individual external magnetic fields $\vec{B}_{i}(i=1,2)$ that determine their respective energy for a given orientation (parallel or anti-parallel to $\vec{B}_{i}$ ). Spin 2 experiences in the presence of spin 1 , in addition to $\vec{B}_{2}$, an additional field that depends on the relative orientation between the two spins. Thus the energy of spin 2 is shifted depending on the orientation of spin 1 (and vice versa). The spatial wavefunctions of the two spins are indicated by $\Phi_{i}\left(\vec{r}_{i}\right)$.

For two electron spins $\left(\gamma_{1,2} \approx e / m_{e}\right.$ with mass $\left.m_{e}\right)$ and assuming $\theta=0$, we find $\Delta \omega \approx 2 \pi \times 2.6 \times 10^{-20} \delta z^{-3} \mathrm{~Hz}$ with $\delta z$ measured in meters. Therefore, for a typical inter-ion separation, $\delta z=5 \mu \mathrm{m}$ the direct magnetic spin-spin interaction amounts to $\Delta \omega \approx 2 \pi \times 0.2$ $\mathrm{mHz}$ and is negligible in typical ion trap settings. This estimate is based on a magnetic dipole-dipole interaction of ions in their ground state. If bringing the ions to a highly excited Rydberg state, one would expect an appreciable interaction between their electric dipoles [Müller et al. 2008, Urban et al. 2009, Gaetan et al. 2009].

In order for the exchange interaction to play a non-negligible role, an overlap of the wavefunctions describing the external, motional degrees freedom of neighbouring ions would be required, or more precisely, the exchange integral $\int r_{12}^{-1}\left[\Phi_{n}\left(\vec{r}_{1}\right) \Phi_{n+1}\left(\vec{r}_{2}\right)\right]\left[\Phi_{n}^{*}\left(\vec{r}_{2}\right) \Phi_{n+1}^{*}\left(\vec{r}_{1}\right)\right]$ would have to have a non-negligible magnitude. Here, the spatial wavefunction of ion number $n(n+1)$ located at position $\vec{r}_{1}\left(\vec{r}_{2}\right)$ is $\Phi_{n}\left(\vec{r}_{1}\right)\left(\Phi_{n+1}\left(\vec{r}_{2}\right)\right)$ and the integration is carried out over all space. However, the root mean square extension of the ground state wavefunction of a trapped ion is given by $\Delta z_{1}=\sqrt{\hbar / 2 m \nu_{1}}=7.1 \times 10^{-8} \times(M f)^{-1 / 2} \mathrm{~m}$ In the case of a ${ }^{9} \mathrm{Be}^{+}\left({ }^{171} \mathrm{Yb}^{+}\right)$ion, one obtains $\Delta z_{1}=24 \mathrm{~nm}(5.4 \mathrm{~nm})$ for $f=1 \mathrm{MHz}$ which typically is three orders of magnitude smaller than the inter-ion separation $\delta z$. Therefore, the exchange interaction can be neglected, too. 


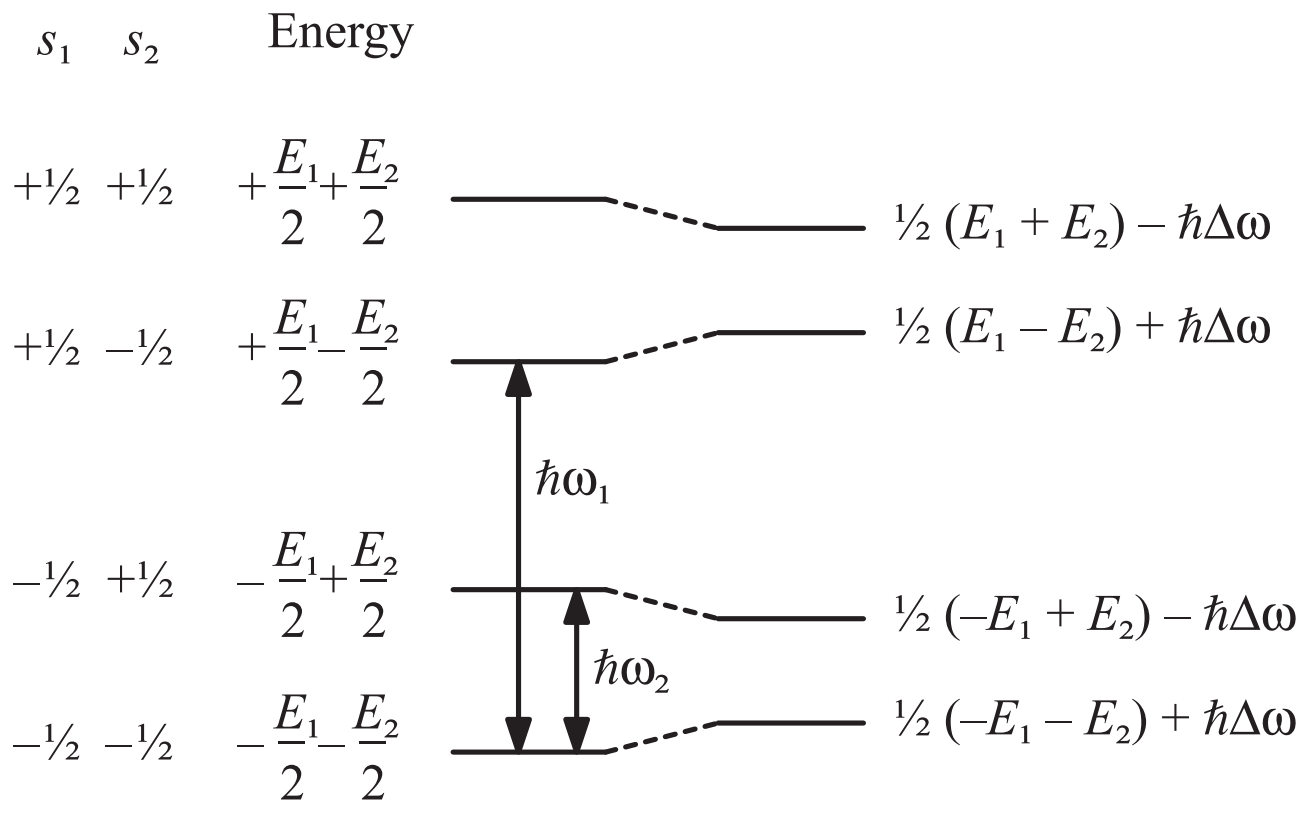

Figure 2. Energy levels of two spins as a function of their orientation. The quantum numbers $s_{i}, i=1,2$ indicate the spin state. The resonance frequencies of non-interacting spins are $\omega_{i}$ while when they interact these resonances are shifted by $\Delta \omega_{i}$.

2.1.2. Spin-spin interaction mediated by the Coulomb force Thus, a direct spin-spin interaction between trapped ions will not be sufficiently strong for a useful implementation of the spin models described above. Instead, we now look for an interaction that is mediated by some other physical mechanism.

Physical picture The applied trapping potential and the Coulomb forces between the ions determine their equilibrium positions. Now, by applying a suitable external field, an additional force is exerted on each ion whose magnitude and/or direction depends on the orientation of the spin of the ion and varies spatially (i.e., depends on the exact location of the ion). If this state-dependent force acts in addition to the trap and Coulomb forces, then a spin flip of ion 1 changes this force and, as a consequence, this ion changes its equilibrium position. Via Coulomb repulsion, this in turn leads to a shift of the equilibrium position of the neighbouring ion 2 that now finds itself exposed to a field of different magnitude and thus changes its internal energy.

This additional field may be a static magnetic field with spatially varying magnitude. The direct interaction between magnetic dipoles (where flipping one spin amounts to a change in the local magnetic field experienced by another nearby spin) is replaced by an indirect interaction where the change in the magnetic field at the location of spin 2 is brought about by a change of its equilibrium position (caused by the spin flip of ion 1) in a spatially varying external field.

For a more quantitative description we consider the force $\vec{F}=\langle(\vec{\mu} \cdot \vec{\nabla}) \vec{B}\rangle$ acting on an atomic magnetic moment $\vec{\mu}=(\gamma \hbar / 2) \vec{\sigma}$ associated with spin $(\hbar / 2) \vec{\sigma}$ in a magnetic field $\vec{B}$. Without loss of generality we restrict the following discussion to the case where 
$\vec{B}=\overrightarrow{B_{0}}+b \hat{z}$ with constant offset field $\overrightarrow{B_{0}}$ and gradient $b$ along the $z$-axis $甘$ such that the force $F_{z}=\left\langle(\gamma \hbar / 2) b \sigma^{(z)}\right\rangle$ changes sign upon flipping the atomic spin $(\langle\rangle$ indicates the expectation value). Using the position dependent spin resonance frequency $\omega=\gamma b z$, the force can be expressed as a function of the change of $\omega$ when moving the ion along the $z$-axis:

$$
F_{z}=(\hbar / 2) \partial_{z} \omega\left\langle\sigma^{(z)}\right\rangle \text {. }
$$

For an ion of mass $m$ confined in a harmonic potential, characterized by angular frequency $\nu$, this linear force that acts in addition to the trapping potential and Coulomb force, shifts the equilibrium position of the ion, upon flipping its spin, by an amount $d_{z}=\mp F_{z} /\left(m \nu^{2}\right)$ from its initial equilibrium position (compare figure 3). Thus, in a classical picture, the ion finds itself on the slope of a harmonic oscillator potential, instead of at the potential minimum, after its spin was flipped. Consequently, it will start to oscillate around the new equilibrium position. In this way the ion's internal dynamics and its motional degrees of freedom can be coupled, even if the radiation causing the spin flip doesn't impart enough linear momentum to excite the ion's motion. This coupling, quantum mechanically described by an effective Lamb-Dicke parameter $\eta_{e f f}=d_{z} / \Delta z$ [Mintert \& Wunderlich 2001, Mintert \& Wunderlich 2003, Wunderlich 2002, Wunderlich \& Balzer 2003], has recently been observed experimentally [Johanning et al. 2009].

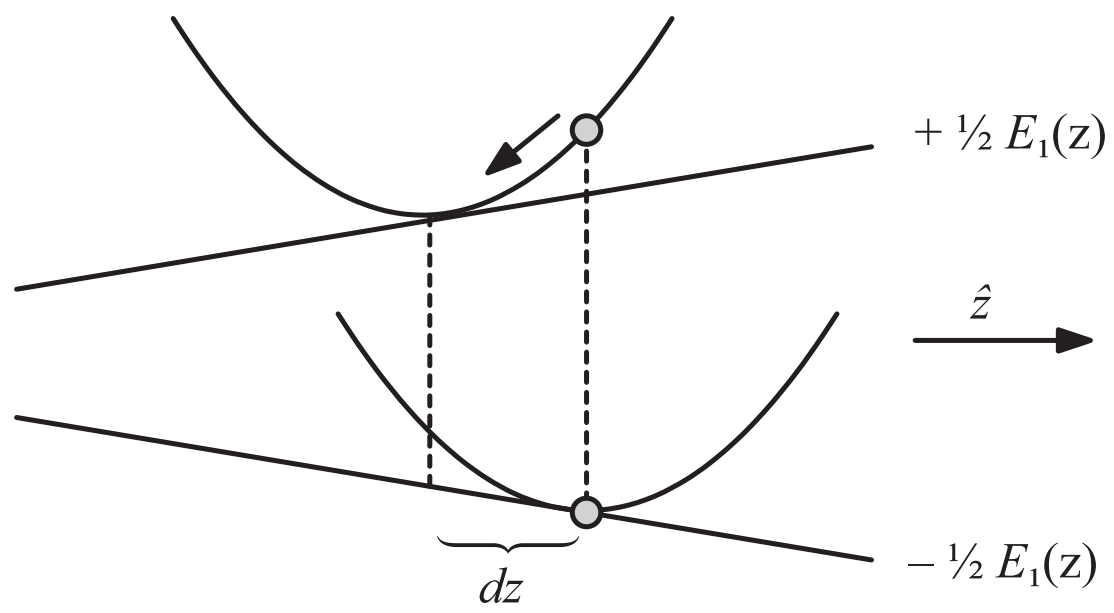

Figure 3. A single ion confined in a potential that is the sum of a harmonic oscillator and a linear term. The sign of the linear term depends on the internal spin state $\left( \pm \frac{1}{2} E_{1}\right)$ of the ion. Upon flipping the ion's spin its motion can be excited.

In order to see how the desired $J$-coupling arises, we consider how an ion's shift of its equilibrium position affects its internal energy. This change of its internal energy is given by

$$
\hbar J=-F_{z} d_{z}=-F_{z}^{2} /\left(m \nu^{2}\right) \propto(b / \nu)^{2} .
$$

As a consequence of one ion's shift of equilibrium position, through Coulomb interaction, the position and energy of neighbouring ions is shifted, too, giving us the desired (indirect) spin-spin interaction (compare figure 4).

$\ddagger$ Such a field may be approximately realized, even though it does not fulfill $\vec{\nabla} \cdot \vec{B}=0$, for example, on the axis of rotational symmetry of a quadrupole field sufficiently far from the point of inflection symmetry. 


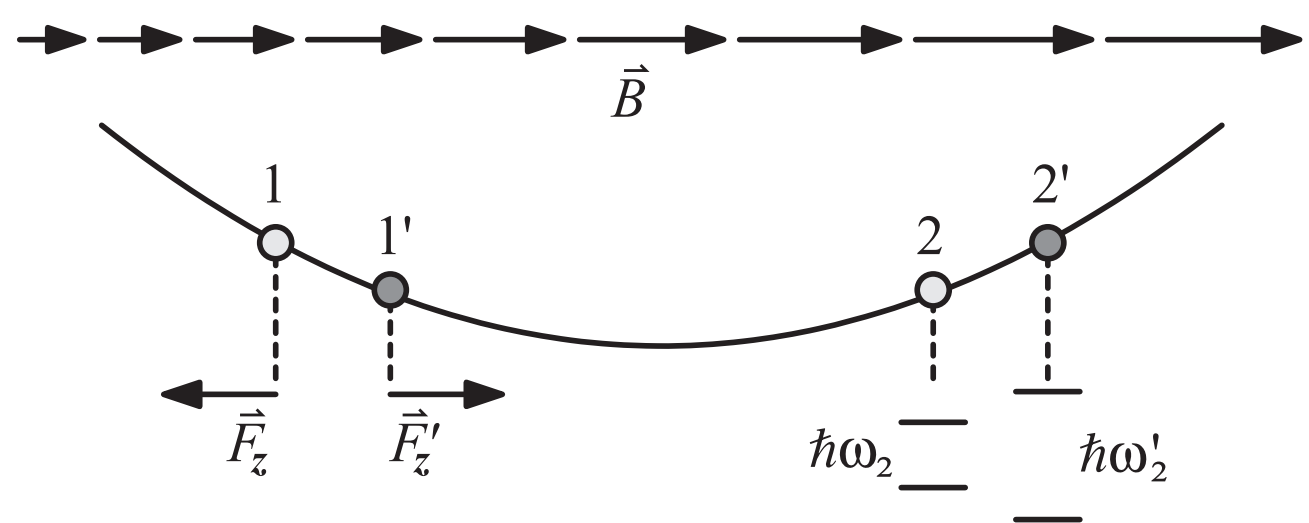

Figure 4. Sketch of two ions confined in a harmonic potential with superimposed spatially varying magnetic field $\vec{B}$ to illustrate spin-spin coupling mediated by Coulomb repulsion. When flipping the spin of ion 1 , the state-dependent force acting on it will change sign, and thus, its equilibrium position will change $\left(1 \rightarrow 1^{\prime}\right)$. Through Coulomb interaction the second ion, too, will change its equilibrium position $\left(2 \rightarrow 2^{\prime}\right)$, and, since it moves in a field gradient, consequently will change its internal resonance frequency from $\omega_{2}$ to $\omega_{2}^{\prime}$.

Calculation of spin-spin coupling After having introduced this general physical mechanism that may be used to induce an effective spin-spin interaction, we will now briefly outline how this may be employed to engineer a variety of interactions between a collection of trapped ions. We consider the internal energies of $N$ ions all of which experience a shift of their internal resonance frequency that, for now, depends on their axial position denoted by $z$, only:

$$
H_{I}=-\frac{\hbar}{2} \sum_{n=1}^{N} \omega_{n} \sigma_{z}^{(n)}-\frac{\hbar}{2} \sum_{n=1}^{N} \partial_{z} \omega_{n} q_{n} \sigma_{z}^{(n)}
$$

Here, $q_{n}$ is the deviation of ion $n$ from its equilibrium position $z_{n}^{(0)}$,

$$
\left.\omega_{n} \equiv \omega\right|_{z_{n}^{(0)}} \quad,\left.\quad \partial_{z} \omega_{n} \equiv \partial_{z} \omega\right|_{z_{n}^{(0)}},
$$

and the expansion of $\omega_{n}$ has been included up to first order[s.

The external (motional) degrees of freedom of $N$ ions are determined by the applied trapping potential and their mutual Coulomb repulsion. Expanding the total potential up to second order gives

$$
V^{(h a r m)}=\frac{m}{2} \sum_{\alpha=1}^{3} \sum_{i, j=1}^{N} A_{\alpha \alpha, i j} q_{\alpha, i} q_{\alpha, j}
$$

where the three spatial degrees of freedom $\alpha=x, y, z$ are uncoupled [James 1998]. The Hessian matrices $A_{\alpha \alpha, i j}$ that characterize the potential experienced by the ions are real valued and symmetric in $i$ and $j$ and are transformed into diagonal matrices $D_{\alpha}$ via $D_{\alpha}=S_{\alpha}^{-1} A_{\alpha}^{(2)} S_{\alpha}$ with eigenvectors $\vec{Q}$.

In terms of the normal coordinates we obtain the following Hamiltonian describing $3 N$

$\S$ We assume a constant field gradient, that is, second and higher order derivatives of the field vanish. However, the expression 10 is still valid even if these derivatives are different from zero as long as the gradient doesn't vary appreciably over the extent $\Delta z$ of an ions spatial wavefunction. 
uncoupled harmonic oscillators [James 1998]:

$$
H_{E}=\sum_{\alpha=1}^{3}\left[\frac{1}{2 m} \sum_{n=1}^{N} P_{\alpha, n}^{2}+\frac{m}{2} \sum_{n=1}^{N} \nu_{\alpha, n}^{2} Q_{\alpha, n}^{2}\right]
$$

where $\nu_{\alpha, n}$ is the angular frequency of the normal vibrational mode $n$ in direction $\alpha=x, y, z$ and the relation between the normal coordinates $Q_{\alpha, n}$ and the local coordinates $q_{\alpha, n}$ is $\vec{Q}_{\alpha} \equiv$ $\left(Q_{\alpha, 1}, \ldots, Q_{\alpha, N}\right)=S_{\alpha}^{-1} \vec{q}_{\alpha}$ with $\vec{q}_{\alpha}=\left(q_{\alpha, 1}, \ldots, q_{\alpha, N}\right)$, furthermore $P_{\alpha, n} \equiv m \dot{Q}_{\alpha, n}$.

The total Hamiltonian includes internal and motional parts $H=H_{I}+H_{E}^{(z)}$, where, for now, we restrict our considerations to the spatial $z$-direction where a field gradient is present. If a magnetic field gradient was present also in the radial direction (which is the case, e.g., for a quadrupole field close to its point of symmetry), then the radial vibrational modes would also contribute to the $J$-coupling. However, this contribution usually is small, since in order to maintain a linear ion string, the angular frequencies $\nu_{x, 1}$ and $\nu_{y, 1}$, characterizing the radial trapping potential, are usually chosen much larger than $\nu_{z, 1}$. Taking into account that the $J$-coupling is proportional to the inverse square of the trap frequency (taking equation 9 as a guideline), we neglect the contribution from transverse vibrational modes for now. In [Porras $\&$ Cirac 2004a, Deng et al. 2005] transverse modes are explicitly considered (see below).

After expressing the local coordinates $q_{n}$ in equation 10 by the normal coordinates, $q_{n}=\sum_{l=1}^{N} S_{n l} Q_{l}$, noting that $Q_{l}=\Delta z_{l}\left(a_{l}^{\dagger}+a_{l}\right), P_{l}=i m \nu_{l} \Delta z_{l}\left(a_{l}^{\dagger}-a_{l}\right)$, and performing a suitable unitary transformation of $H, \tilde{H}=U^{\dagger} H U$, with

$$
U \equiv \exp \left(-\frac{i}{\hbar} \sum_{n, l=1}^{N} \Delta z_{l} \varepsilon_{n l} \sigma_{z}^{(n)} P_{l}\right)
$$

we obtain [Wunderlich 2002]

$$
\tilde{H}=-\frac{\hbar}{2} \sum_{n=1}^{N} \omega_{n} \sigma_{z}^{(n)}+\hbar \sum_{n=1}^{N} \nu_{n} a_{n}^{\dagger} a_{n}-\frac{\hbar}{2} \sum_{\substack{i, j=1 \\ i<j}}^{N} J_{i j l} \sigma_{z}^{(i)} \sigma_{z}^{(j)} .
$$

where the vibrational motion and the internal states are now decoupled $\left(a_{n}^{\dagger}, a_{n}\right.$ are the creation and annihilation operator of vibrational mode $n$ ). The spin-spin coupling constants are given by

$$
J_{i j} \equiv \sum_{n=1}^{N} \nu_{n} \varepsilon_{i n} \varepsilon_{j n}
$$

where the dimensionless constants

$$
\varepsilon_{i n} \equiv \frac{\Delta z_{n} \partial_{z} \omega_{i}}{\nu_{n}} S_{i n}
$$

have been introduced. The numerator of the fraction on the right-hand-side of equation 16 gives the change of the internal energy (or frequency) that ion number $i$ undergoes when its position is shifted by an amount equal to the extension of the ground state wavefunction, $\Delta z_{n}=\sqrt{\frac{\hbar}{2 m \nu_{n}}}$ of vibrational mode $n$. This is set in relation to the energy (frequency) of a phonon of mode $n$. The matrix element $S_{i n}$ gives the scaled deviation of ion $i$ from its equilibrium position when vibrational mode $n$ is excited. Thus, $\varepsilon_{i n}$ tells us how strongly ion number $i$ couples to vibrational mode $n$ when its spin is being flipped. The sum in equation 15 extends over all vibrational modes, since the local deviation, $q_{n}$ of ion $i$ from its equilibrium position potentially (if $S_{i n}$ is different from zero) excites all vibrational modes of the ion string. 
Noting that $\nu_{n} \propto \nu_{1}$ and $F_{z, i} \propto \partial_{z} \omega_{i}$ and plugging the expression for $\Delta z$ into equation 16 we find from equation 15 that

$$
\begin{aligned}
J_{i j} & \propto \frac{F_{z, i} F_{z, j}}{\nu_{1}^{2}} \\
& \propto\left(\frac{b}{\nu_{1}}\right)^{2}
\end{aligned}
$$

in agreement with the result 9 that was found from a simple model based on classical considerations (here, $\nu_{1}$ is the angular frequency of the centre-of-mass mode of $N$ ions). Going from the first to the second line in equation 17, we have assumed a force caused by a constant magnetic field gradient causing a linear Zeeman shift of the spin states of ions $i$ and $j$.

This spin-spin coupling is only weakly sensitive to thermal excitation of the ion string. Thus, usually simple Doppler cooling will be sufficient to avoid unwanted thermal effects on the coupling constants $J_{i j}$ [Loewen \& Wunderlich 2004]. Detailed calculations on this aspect will be published elsewhere.

In [Ospelkaus et al. 2008] it is shown how an oscillating magnetic field generated in a micro-structured surface trap may be used to induce spin-coupling between ions and how this could be employed to carry out quantum gates.

Spin-spin interaction created by an optical force The $J$-coupling described above may also be created by a state-dependent optical force (instead of the magnetic force assumed above) induced by an off-resonant standing laser wave [Porras \& Cirac 2004a, Deng et al. 2005]. Applying state-dependent forces (e.g., optical or magnetic forces) along different spatial directions, combined with various trapping conditions, a variety of different coupling Hamiltonians of the general form

$$
H_{s}^{(i o n)}=-\frac{1}{2} \sum_{\alpha=x, y, z} \sum_{i>j} J_{i j}^{(\alpha)} \sigma_{i}^{\alpha} \sigma_{j}^{\alpha}-\sum_{i} \sigma_{i}^{\alpha} \vec{B}_{i}^{\alpha^{\prime}}
$$

may be realized [Porras \& Cirac 2004a, Deng et al. 2005].

In particular, in [Deng et al. 2005] it is shown how state-dependent forces that act in the radial direction of a 1-d ion string may be exploited to simulate a transverse quantum Ising model and an XY-model, either with Coulomb crystals confined by a global potential, or linear arrays of microtraps where each ion is confined in its own, externally controlled potential well. It is demonstrated (by means of numerical and analytical calculations) under which conditions quantum phase transitions should be observable with 1-d strings of trapped ions, and what the expected characteristic features of these transitions are, as compared to the usual Ising and XY-models where nearest-neighbour interaction only is considered.

2.1.3. Engineering the spin-spin coupling A straight forward way to apply a magnetic gradient that induces the long-range $J$-coupling described above in a linear Coulomb crystal is the use of current carrying coils in an anti-Helmholtz type arrangement, mounted such that their axis of rotational symmetry coincides with the trap $z$-axis. The field generated by these coils is easily calculated analytically and gradients up to several tens $\mathrm{T} / \mathrm{m}$ are attainable with coils with a diameter of typically $1 \mathrm{~mm}$. Some examples for possible configurations and computed examples for $J$-coupling constants are presented in [Wunderlich \& Balzer 2003, Wunderlich et al. 2005, Wunderlich \& Wunderlich 2009]. Such coils may also be integrated into micro-structured ion traps that are currently being 
developed. Here, larger gradients are realistic (several hundreds of $\mathrm{T} / \mathrm{m}$ ) [Brüser n.d.]. When using current-carrying structures for generating magnetic fields, the currents, and thus the $J$-coupling, can be turned on and off as required, however, the stability of the magnetic field needs attention to avoid dephasing of spins. Alternatively, permanent magnets may be used [Johanning et al. 2009].

Global trap potential If the ions are confined in one common trapping potential, then the strength of the $J$-coupling may be controlled globally, that is, all coupling constants may be varied together by adjusting the gradient $b$ and/or the secular trap frequency $\nu_{1}$. In a linear Paul trap the trap frequency along the $z$-axis of a linear trap is adjustable by applying a suitable voltage to the end cap electrodes, while the radial trapping frequency can be adjusted by varying the amplitude of the applied rf trapping field. If the gradient $b$ is generated by electrical currents, then these currents can be varied, and turning the magnetic field on and off using a desired envelope allows for simultaneous switching of all coupling constants. If permanent magnets are used, and their position is fixed along the $z$-axis (typically generating a quadrupole field), the ions may be moved collectively along the $z$-axis to change the gradient they experience. Moving the ions in a linear Paul trap is achieved by applying suitable voltages to the electrodes that are responsible for the axial confinement. Even an alwayson interaction (that arises, for instance, when using permanent magnets to generate a field gradient) used in conjunction with coherent manipulation of individual spins maybe employed such that prescribed entangled states can be generated in a time-optimal way [Fisher n.d.]. for the axial confinement. Even an always-on interaction (that arises, for instance, when using permanent magnets to generate a field gradient) used in conjunction with coherent manipulation of individual spins maybe employed such that prescribed entangled states can be generated in a time-optimal way [Fisher n.d.].

Locally variable trap potential The advent of micro-structured traps brings with it the possibility to attain control over local trapping potentials and thus, of engineering the $J$-coupling locally. For example, employing a linear Paul trap with dc electrodes that are segmented such that individual electrodes have an axial extension of the order of the inter-ion separation allows for shaping the local electrostatic potential such that long-range coupling is strongly suppressed and essentially only uniform nearest-neighbour coupling exists [Hugh \& Twamley 2005].

Furthermore, micro-structured traps with segmented electrodes allow for detailed local sculpting of $J$-coupling constants. This can be useful to generate effective time evolution operators making it possible to reach a desired entangled state in a single time evolution step without the need for refocussing. Examples how to generate cluster states in this way are given in [Wunderlich \& Wunderlich 2009].

A possible drawback of the use of small electrodes is that this usually implies an equally small distance between ions and nearby surfaces (i.e., typically of order $10 \mu \mathrm{m}$ ) which leads to high heating rates of the ions' secular motion, and thus limits the useful time available for coherent dynamics [Turchette et al. 2000]. Using ion traps embedded in a cryogenic environment considerably reduces this heating rate [Deslauriers et al. 2006, Labaziewicz et al. 2008], and therefore, may provide a feasible approach to using small electrode structures. A micro-fabricated linear surface trap cooled to $4 \mathrm{~K}$ that includes current-carrying structures for generating a magnetic field gradient was recently demonstrated [Wang et al. 2008]. The magnetic gradient was used to address individual ${ }^{88} \mathrm{Sr}^{+}$ions in frequency space on an optical transition between Zeeman states of the electronic ground state $5 \mathrm{~S}_{1 / 2}$ and the metastable state 
$4 \mathrm{D}_{5 / 2}$, respectively.

Segmented ion traps also allow for transporting ions between different trap locations [Wineland, Monroe, Itano, Leibfried, King \& Meekhof 1998, Kielpinski et al. 2002, Rowe et al. 2002, Home \& Steane 2006, Hensinger et al. 2006, Schulz et al. 2006, Reichle et al. 2006, Hucul et al. 2008, Huber et al. 2008]. For moving ions, the required electrode structures may be considerably larger, typically of order $100 \mu \mathrm{m}$, thus helping to reduce unwanted heating. The transport of ions over small distances in segmented ion traps gives a further handle to engineer effective couplings by letting only a subset of ions interact at a time in a prescribed way. This could be used, for example, to generate two-dimensional cluster states using a one-dimensional linear Coulomb crystal [Wunderlich \& Wunderlich 2009].

A possible architecture for a two-dimensional surface trap array exploiting magnetic field-gradient induced spin-spin coupling as described above was proposed in [Chiaverini \& Lybarger 2008]. In [Clark et al. 2008] confinement of ${ }^{88} \mathrm{Sr}^{+}$ions in a 2-d ion trap array with individual sites spaced apart by about $1.6 \mathrm{~mm}$ is reported. Even though a trap array with such a large inter-ion spacing does not allow for a useful spin coupling between the ions, it is nevertheless useful as a proof-of-principle and makes possible the investigation of the scaling behaviour of this trap array. It turns out that scaling down of the layered rf lattice trap alone is unlikely to be a promising route to achieve useful $J$ - coupling. It appears that further modifications of this trap design are useful to ensure low enough secular frequencies for sufficiently strong $J$-coupling (compare equation 17 ).

Recently, two-dimensional and linear arrays of Penning traps that confine individual electrons have been proposed as a promising physical system for quantum computation and quantum simulations. Here, the physical origin of the $J$-coupling again is a force that depends on an electron's spin state and is caused by a magnetic field gradient and, as in ion traps, the Coulomb force between electrons mediates the spin-spin coupling [Ciaramicoli et al. 2005, Ciaramicoli et al. 2007, Ciaramicoli et al. 2008]. Thus, various couplings as in equation 3 with variable range could be realized with trapped electrons and used, for instance, to investigate quantum phase transitions.

2.1.4. Simulating an effective transverse field An effective transverse magnetic field can be simulated by driving transitions between the spin states of ion $i$ employing a radio frequency or optical field resonant with this ion's qubit transition [|:

$$
\begin{aligned}
H_{t} & =-\hbar \Omega_{R} \cos \left(\omega^{(i)} t+\phi^{\prime}\right) \sigma_{x}^{(i)} \\
& =-\frac{\hbar}{2} \Omega_{R}\left(\sigma_{+}^{(i)}+\sigma_{-}^{(i)}\right)\left(e^{i \omega^{(i)} t+\phi^{\prime}}+e^{-i \omega^{(i)} t+\phi^{\prime}}\right) .
\end{aligned}
$$

Here, $\Omega_{R}$ is the Rabi frequency that for a linearly polarized rf field $B_{x} \cos \left(\omega_{x} t+\phi^{\prime}\right)$ reads $\Omega_{R}=\gamma B_{x} / 2$ and the resonance condition is $\omega_{x}=\omega^{(i)}$.

First transforming $H_{t}$ by $U$, as was done above for the Hamiltonian $H$ (equation [15], then carrying out a transformation into an interaction picture with respect to $-\frac{\hbar}{2} \sum_{n=1}^{N} \omega_{n} \sigma_{z}^{(n)}+$ $\hbar \sum_{n=1}^{N} \nu_{n} a_{n}^{\dagger} a_{n}$ [Wunderlich 2002], expanding the resulting Hamiltonian up to first order in $a^{\dagger}$ and $a$, neglecting terms that contain $\varepsilon_{i n}$, and applying the rotating wave approximation, one obtains for spin $i$

$$
\tilde{H}_{t}^{(i)}=-\frac{\hbar}{2} \Omega_{R}\left(\cos \phi \sigma_{x}^{(i)}-\sin \phi \sigma_{y}^{(i)}\right)
$$

|| Optical fields may be used to drive transitions in the optical range, but also in the RF range by applying a twophoton Raman excitation

I In [Deng et al. 2005] the effects of residual spin-phonon coupling caused by the effective transverse field are calculated 
when the rf field or optical field is on resonance. Allowing for a detuning $\omega_{r f}-\omega^{(i)}$ between the applied field and the spin flip resonance frequency introduces a term proportional to $\left(\omega_{r f}-\omega^{(i)}\right) \sigma^{(z)}$ on the rhs of equation 21. Thus, by driving spin transitions of individual ions, a variety of additional local effective magnetic fields ${\overrightarrow{B_{n}}}^{\prime} \propto\left(\cos \phi_{n},-\sin \phi_{n},\left(\omega_{r f}-\omega^{(n)}\right)^{T}\right.$ in Hamiltonian 3 may be simulated. In particular, by choosing the phase $\phi^{\prime}$ of the rf field such that $\phi=0$ and fulfilling the resonance condition for all ions, a global transverse field $B_{x}^{\prime}$ for the transverse Ising model 4 is implemented.

2.1.5. Spin-boson model The spin-boson model ([Leggett et al. 1987, Weiss 1999] and references therein) is the starting point for many theoretical investigations modeling, for instance, the decoherence of a variety of physical system. It describes the dynamics of a spin$1 / 2$ coupled to a bath of harmonic oscillators that represent the spin's environment. Thus, the spin-boson Hamiltonian contains a term proportional to $\sigma_{z}$ (describing the spin's energy), a term proportional to $\sigma_{x}$ (describing spin flips), a term describing the harmonic oscillators, $\hbar \sum_{l} \nu_{l}\left(a_{l}^{\dagger} a_{l}+1 / 2\right)$, and finally a term coupling the spin to the oscillators

$$
\frac{\hbar}{2} \sigma_{z} \sum_{l} C_{l} Q_{l}
$$

where $Q_{l}$ is the position coordinate of the $n$-th harmonic oscillator while $C_{l}$ describes the coupling strength between this oscillator and the spin. We note that all these ingredients are already present in the formulae presented above: Picking out a particular ion $i$, equation 10 may be rewritten as

$$
\begin{aligned}
H_{I}^{i} & =-\frac{\hbar}{2} \omega_{i} \sigma_{z}^{(i)}-\frac{\hbar}{2} \sigma_{z}^{(i)} \sum_{n=1}^{N} \partial_{z} \omega_{i} q_{n} \\
& =-\frac{\hbar}{2} \omega_{i} \sigma_{z}^{(i)}-\frac{\hbar}{2} \sigma_{z}^{(i)} \sum_{l=1}^{N} \partial_{z} \omega_{i}\left(\sum_{n=1}^{N} S_{n l}\right) Q_{l}
\end{aligned}
$$

and we can set $C_{l}=\partial_{z} \omega_{i}\left(\sum_{n=1}^{N} S_{n l}\right)$ to obtain in equation 22 the coupling between spin $i$ and the harmonic oscillators represented by the collective vibrational modes of a string of $N$ ions. The term proportional to $\sigma_{x}$ is obtained by driving transitions between the two spin orientations (see below, eq. 21).

Now the question arises how to physically single out a particular spin $i$ from the sum over all spins in the second term of $H_{I}$ (equation 10) such that we are left with $H_{I}^{i}$ in equation 23. This could be done by exerting a state-dependent force only on ion $i$ as is shown in [Porras et al. 2008]. Porras et al. detail how laser beam(s) focussed onto one particular ion give rise to optical forces that induce the required coupling. Furthermore, it is shown how phonon baths characterized by a variety of spectral densities may be simulated and how effects caused by the finite size of the ion crystal may be observed.

\subsection{Phase transition}

2.2.1. Quantum transverse Ising model The quantum transverse Ising model (TIM, equation 4. displays a phase transition already in one dimension [Sachdev 1999], even at zero temperature, between an ordered state and a disordered state upon varying $B_{x}^{\prime} / J$. Milburn et al. [Milburn 1999, Milburn et al. 2000] proposed an implementation of a unitary map closely related to the TIM using Raman laser pulses. In [Porras \& Cirac 2004a] it is proposed how to observe this quantum phase transition (QPT) with trapped ions interacting with a 
standing laser wave that creates a long-ranged spin-coupling as outlined above (section 2.1.2). Barjaktarevic et al. [Barjaktarevic et al. 2005] proposed to implement a unitary map that displays a QPT belonging to the same universality class as the transverse Ising model.

In order to get a qualitative picture of such a phase transition we first consider the collection of spins described by $H_{T I}$ (equation 4) in the limit where the effective transverse field, $B_{x}^{\prime}$ assumes a finite value, but the mutual coupling $J$ between spins vanishes, $B_{x}^{\prime} \neq$ $0=J$, such that, at zero temperature, all spins are oriented along the $x$-direction and the groundstate $|0\rangle=\prod_{i}|\rightarrow\rangle_{i}=\prod_{i}\left(|\uparrow\rangle_{i}+|\downarrow\rangle_{i}\right)$ of the system is a product of eigenstates of $\sigma_{i}^{x}$. Consequently, $\left\langle 0\left|\sigma_{i}^{z} \sigma_{j}^{z}\right| 0\right\rangle=\delta_{i j}$, that is, the orientation of different spins with respect to the the $z$-axis is uncorrelated. On the other hand, if the spin-spin coupling doesn't vanish, $B_{x}^{\prime} \gg|J|>0$, the spins will acquire a tendency to align along the $z$-axis. Thus, the ground state of the system will start to exhibit non-vanishing, but short ranged correlations between spins located at sites $r_{i}$ and $r_{j}$, respectively, with a characteristic length scale $\xi$ [Sachdev 1999]

$$
\left\langle 0\left|\sigma_{i}^{z} \sigma_{j}^{z}\right| 0\right\rangle \propto e^{-\left|r_{i}-r_{j}\right| / \xi} .
$$

Here, $|0\rangle$ is the system's ground state when $B_{x}^{\prime} \gg|J| \neq 0$.

Now, we turn to the limit $B_{x}^{\prime} \ll J>0$. If $B_{x}^{\prime}$ vanishes completely, then an infinite system of spins possesses two degenerate ferromagnetic ground states where all spins are either parallel or anti-parallel to the $z$-axis, and the total magnetization of the system is $M_{0}$. Turning on a small field $B_{x}^{\prime} \ll J$ pointing in the $x$-direction will tend to reorient some of the spins along the $x$-direction (classically, their magnetic moments will start to precess around the $x$-direction, thus flipping spins between the two possible $z$-orientations). The ground state of the system, $|0\rangle$ is still close to the $B_{x}^{\prime}=0$ case and is obtained from a perturbative expansion in the small parameter $B_{x}^{\prime} / J$. One finds $\left\langle 0\left|\sigma_{i}^{z}\right| 0\right\rangle=M / M_{0}$ where $M<M_{0}$ is now the spontaneous magnetization of the system. Again calculating the correlation of the orientation of different spins in the $z$-direction for this ground state gives [Sachdev 1999]

$$
\lim _{\left|r_{i}-r_{j}\right| \rightarrow \infty}\left\langle 0\left|\sigma_{i}^{z} \sigma_{j}^{z}\right| 0\right\rangle=\left(\frac{M}{M_{0}}\right)^{2} .
$$

The expressions 24 and 25 for the spin correlation are incompatible in the sense that a continuous variation of $B_{x}^{\prime} / J$ between the two limiting cases considered above doesn't allow for an analytical connection between the two expressions on the right-hand-sides of equations 24 and 25. Thus, there must exist a critical value of $B_{x}^{\prime} / J$ where the correlation changes between these two expressions. This is the case even at zero temperature being indicative for a quantum phase transition.

An experimental realization of the proposal by Porras and Cirac [Porras \& Cirac 2004a] with two trapped and laser cooled ${ }^{25} \mathrm{Mg}^{+}$ions was recently reported by Friedenauer et al. [Friedenauer et al. 2008]. Here, the coupling $J$ between spins is induced by a state-dependent ac Stark shift caused by a "walking" standing wave. Two hyperfine states of the electronic ground state of each ${ }^{25} \mathrm{Mg}^{+}$ion served as an effective spin-1/2 with eigenstates $|\downarrow\rangle$ and $|\uparrow\rangle$. In brief, the experimental procedure was as follows: Optical pumping is employed to first initialize the two ions in state $|\downarrow, \downarrow\rangle$. Resonantly driving the transition between the two hyperfine states with RF radiation simulates an effective transverse field $B_{x}^{\prime}$ (section 2.1.4) and allows for preparation of the initial paramagnetic state $|\rightarrow, \rightarrow\rangle$ (both spins are oriented along the effective $B_{x}^{\prime}$ field). Then, the $J$-coupling between the two spins is ramped up adiabatically from zero to a desired value such that the ratio $J / B_{x}^{\prime} \leq 5.2$. For $J \gg B_{x}^{\prime}$ one expects that the two spins are found in a ferromagnetic state, that is, aligned along the $z$-axis. In general, the new ground state will be a superposition of the states $|\downarrow, \downarrow\rangle$ and $|\uparrow, \uparrow\rangle$ which 
is indeed found experimentally. In a further experiment the two spins were prepared initially anti-parallel to the effective $B_{x}^{\prime}$ field, an excited paramagnetic state. Turning on the spin-spin coupling adiabatically then resulted in an excited state in the presence of strong $J$-coupling $\left(J / B_{x}^{\prime} \gg 1\right)$ : the anti-ferromagnetic state $|\uparrow, \downarrow\rangle+|\downarrow, \uparrow\rangle$. In both cases the entanglement of the final state was proven experimentally.

2.2.2. Scalar fields Classical phase transitions between spatial configurations of ions confined in a linear or ring shaped trap have been investigated experimentally and theoretically [Waki et al. 1992, Raizen et al. 1992, Birkl et al. 1992, Dubin 1993, Schiffer 1993, Drewsen et al. 1998, Kjærgaard \& Drewsen 2003, Morigi \& Fishman 2004a, Morigi \& Fishman 2004b, Fishman et al. 2008]. Retzker et al. [Retzker et al. 2008] suggest to explore a phase transition between a linear and a zig-zag configuration [Fishman et al. 2008] in the quantum regime, that is, with the ion string cooled close to its vibrational ground state. The ion trap parameters are chosen such that a linear Coulomb crystal forms (i.e., the confinement in the axial direction is weaker than in the radial direction), and, in addition, $\nu_{y, 1} \gg \nu_{x, 1}$ such that one radial degree of freedom is essentially frozen out after laser cooling, but the other may be employed for quantum simulations. Linear Paul traps as well as Penning traps where the ions are confined on the axis of rotational symmetry [Powell et al. 2002] are well suited for this purpose.

For $\nu_{1, x}$ below a critical value $\nu_{1, x}^{c}$, a zig-zag configuration of the ions will form with two degenerate states. It is shown in [Retzker et al. 2008] that coherent tunneling between these two states maybe observed and used for interferometry. Furthermore, by adjusting $\nu_{1, x}$ around $\nu_{1, x}^{c}$ the parameters of a Hamiltonian describing the ions' motional excitation along the $x$-direction may be determined. This Hamiltonian describes at the same time a nonlinear scalar field and by adjusting $\nu_{1, x}$ the effective mass of this field may be varied, in particular in the vicinity of a phase transition. An attractive feature of this proposal is that global adjustment of the radial trapping frequency $\nu_{1, x}$ is sufficient and no local control over the trapping potential is needed.

\subsection{Neural Network}

In [Pons et al. 2007, Braungardt et al. 2007] it was shown that a linear Coulomb crystal of laser cooled trapped ions may be used to implement a neural network (NN) - according to the Hopfield model [Hopfield 1982] - allowing for robust storage of classical information, and furthermore, that such a NN may be employed for error-resistant quantum computation. We will first briefly introduce some features of the Hopfield model (see, e.g., [Rojas 1996] chapter 13 and references therein) and then outline how some features may be realized with trapped ions.

A neural network according to the Hopfield model consists of $N$ nodes (corresponding to neurons) that are all mutually interconnected and we indicate the (synaptic) strength of the connection between node $i$ and node $j$ by $J_{i j}$. This connection is symmetric, that is $J_{i j}=J_{j i}$ and the nodes do not feed back to themselves, $J_{i i}=0$. Below we will see what exactly the meaning of the coupling strengths $J_{i j}$ is. Each node can assume one of two possible states which we label by $s_{i}= \pm 1$ (compare figure 5). When modeling neurons, this would indicate whether the neuron is "firing" (a sudden change of a biologically relevant potential difference) or in a quiescent state at a given time. Furthermore each node is characterized by a threshold $h_{i}$ that, in addition to the couplings $J_{i j}$, influences its response to the the input from other nodes.

A characteristic feature of a Hopfield NN is that it can store information distributed over all nodes. Thus, it is robust against errors in individual nodes or even the loss of 
some nodes and against thermal noise. The dynamics of the NN, being determined by the strengths of interactions between all nodes, brings the system back to one out of a selection of particular states (that we call patterns) once this state was disturbed. This feature may be taken advantage of for pattern recognition: When the $\mathrm{NN}$ is presented with some input state, which is done by initializing all nodes with a given value, then the $\mathrm{NN}$ evolves into the pattern that is was trained to recognize that is closest to this initial state. So how does the NN dynamically evolve?

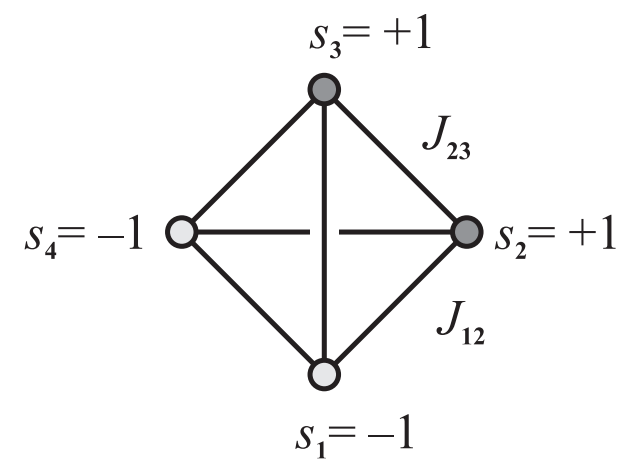

Figure 5. Illustration of a neural network that consists of four nodes according to Hopfield [Hopfield 1982]. Each node may exist in one out of two states indicated by $s_{i}= \pm 1$, $i=1,2,3,4$. The nodes are all interconnected with $J_{i j}=J_{j i}$. Here, the network forms a pattern $\vec{\xi}=(-1,+1,+1,-1)^{T}$. (Note that the number $p$ of patterns that can be stored by a Hopfield network is about $p=0.14 N$ where $N$ is the number of nodes).

We start at time $t$ with an initial global state of the NN labeled by the $N$-component vector $\vec{s}$ where each local node is characterized by $s_{i}$. Then the state of each node at time $t+\delta t$ is determined by the sign of the activation function $A$ at time $t+\delta t$ :

$$
s_{i}(t+\delta t)=\operatorname{sgn}(\mathrm{A}(\mathrm{t}+\delta \mathrm{t})),
$$

where

$$
A(t+\delta t)=\sum_{j \neq i} J_{i j} s_{j}(t)+h_{i} .
$$

This means that at time $t+\delta t$, node $i$ will be firing $\left(s_{i}=+1\right)$ or in a quiescent state $\left(s_{i}=-1\right)$ depending on the state of all other nodes at time $t$, weighted with the strength $J_{i j}$, and the individual threshold $h_{i}$. One of the two following alternative model assumptions is usually made: The updating of node $i$ may either occur sequentially, one node after the other, or in parallel all at the same time.

We now define the cost function

$$
H_{H}(\vec{s})=-\frac{1}{2} \sum_{i, j(i \neq j)} J_{i j} s_{i} s_{j}-\sum_{i} h_{i} s_{i}
$$

and it can be shown [Rojas 1996] that an evolution of the network according to the dynamical rule for sequential updating 26 (together with the definition 27) leads to a monotonous decrease of $H_{H} \uplus$. This means that flipping spins (in agreement with the rules) drives the system towards local or global minima in the "landscape" defined by $H_{H}$ over the space of $\vec{s}$.

+ In this brief account we consider the system at zero temperature only 
Immediately we realize that the expression defining $H_{H}$ is formally equivalent to the spin Hamiltonian $H_{s}$ (equation 3) considering the case of spin-1/2 particles with $J_{i j}^{(x)}=0=J_{i j}^{(y)}$. As noted at the beginning of the section on spin models, further simplifications of $H_{s}$, namely uniform $J$-coupling, only nearest-neighbour interaction, and no transverse field lead to the Ising model (equation 1). When considering the Ising model one usually assumes that all coupling constants are positive or all are negative and thus arrives at a limited set of minima (e.g., for $J>0$ all spins are all either up or down). However, here the couplings $J_{i j}$ are not restricted to being all positive or all negative, thus allowing for a rich "energy landscape" with many minima.

Our goal is to "teach" the NN a specific pattern, $\vec{\xi}, \xi_{i}= \pm 1$ towards which the NN is supposed to relax, if it is displaced from this pattern, or if presented with a similar input pattern. In other words, this pattern must correspond to a minimum of $H_{H}$. Where exactly this minimum occurs, that is, for which particular configuration $\vec{s}$, is determined by the choice of the interaction strengths $J_{i j}$. Therefore, if one wants the NN to "remember" a particular pattern $\vec{\xi}$, (i.e., create a minimum of $H_{H}$ for a specific arrangement $\vec{\xi}$ of the nodes), the $J$-couplings need to be adjusted accordingly. At first sight (and probably still at second) it is not obvious how a set of $J$-couplings may be found that creates a minimum of $H_{H}$ for a desired pattern and thus makes the NN evolve towards this pattern. It turns out that using Hebb's rule [Hebb 1949, Mezard et al. 1987] to determine the values of the couplings $J_{i j}$ gives just the right set of $J$-couplings.

In order to memorize a pattern $\vec{\xi}$, each $J_{i j}$ is modified according to Hebb's rule of learning, such that

$$
J_{i j}^{\text {new }}=\lambda J_{i j}^{\text {old }}+\epsilon \xi_{i} \xi_{j} .
$$

When starting with an "empty" memory, then $J_{i j}^{\text {old }}=0$. In the original Hopfield model $\lambda=1=\epsilon$; another choice is $\epsilon=1 / N$. With the set of interconnections, $J_{i j}$ determined by equation 29 the $\mathrm{NN}$, upon turning on its dynamics, will eventually end up in the memorized pattern $\vec{\xi}$. If the $\mathrm{NN}$ is supposed to memorize $p$ patterns, $\vec{\xi}^{\mu}$ with $\mu=1,2,3, \ldots, p$ instead of just a single pattern, then the learning rule is modified, namely

$$
J_{i j}=\epsilon \sum_{\mu=1}^{p} \xi_{i}^{\mu} \xi_{j}^{\mu} .
$$

Here, we start with an empty memory, i.e. initially $J_{i j}=0 \forall i, j$. After fixing the strength of the pairwise $J$-coupling in this way, the patterns are "memorized" by the NN, and, when presented with some initial state, the NN will evolve towards a minimum of $H_{H}$ associated with one of these patterns. The upper limit for the number of patterns that such a network may faithfully recall via its associative memory is about $0.14 N$ [Rojas 1996].

Now, it will be discussed how a linear Coulomb crystal of trapped ions may be employed to realize a Hopfield-type NN. We have already noted the similarity between $H_{H}$ (equation 28) on one hand and the two spin Hamiltonians $H_{s}^{(a)}$ (equation 3) and $H_{s}^{(i o n)}$ (equation 18) for $J_{i j}^{(x)}=0=J_{i j}^{(y)}$ on the other. A further similarity can be seen between equation 30 , defining the synaptic strengths in the Hopfield model and equation 15 where the ion spin coupling constants are given. We rewrite equation 15 using the expression 16 assuming a constant field gradient and obtain

$$
J_{i j}^{(i o n)}=\frac{\hbar}{2 m} \partial_{z} \omega \sum_{n=1}^{N} \frac{1}{\nu_{n}^{2}} S_{i n} S_{j n}
$$

where the matrix elements $S_{\text {in }}$ may assume positive and negative values, however usually different from \pm 1 as in the generic Hopfield model. Also, the sum in equation 31 extends 
over all $N$ vibrational modes instead of $p$ patterns, and the contribution to $J_{i j}^{(i o n)}$ from each vibrational mode $n$ is weighted by the square of the respective vibrational frequency $\nu_{n}$. Despite these differences, it is possible to store spin patterns with trapped ions in a robust way.

What are the patterns, based on the learning rule 31, that can be stored by a trapped ion neural network? The orientation of a specific ionic spin $i$ in a pattern $\vec{\xi}$ of spin orientations is determined by the sign of $S_{i n}$ for a given vibrational mode $n$. In case of the COM mode, for example, we have $S_{i n}>0$ (or $S_{i n}<0$ ) $\forall i$ which means that in the two associated patterns all spins are either pointing up or all down.

Numerical simulations [Pons et al. 2007, Braungardt et al. 2007] show that, in order to robustly store a pattern of spin orientations it is necessary to adjust the trapping potential such that the vibrational frequencies $\nu_{1}$ and $\nu_{2}$ (that appear in the learning rule 31) are nearly degenerate. This can be achieved by using ion traps with segmented electrodes where the Hessian of the trapping potential can be tuned by applying suitable voltages to individual segments [Hugh \& Twamley 2005, Wunderlich \& Wunderlich 2009]. The robustness of the ion NN has been quantified by Monte Carlo simulations for a string of $40{ }^{40} \mathrm{Ca}^{+}$ions trapped such that the two lowest vibrational modes characterized by $\nu_{1}$ and $\nu_{2}$ are nearly degenerate. For this purpose, a spin pattern $\overrightarrow{\xi^{\mu}}, \mu=1,2$ corresponding to either one of the two modes is prepared as the initial condition of the ions' spin orientations. Then a fixed number $r$ of spins are randomly selected and their state is inverted. This is followed by the time evolution of the ion string until an equilibrium state is reached. The probability of the ion string for ending up in the initial pattern is found to be more than $97 \%$ even when up to eight randomly selected spins are initially in the wrong state (i.e., they are not matching the desired pattern). Thus, even with $20 \%$ of the spins being flipped, the ion string returns towards a desired pattern.

So far we have looked at the robustness of a Hopfield-type neural network where each node consists of a single ion and which is used for classical information storage. For a quantum neural network, the elementary unit for information processing is, instead of a single spin-1/2 with two eigenstates corresponding to $|0\rangle$ and $|1\rangle$, a pattern $\overrightarrow{x i}$ of $N$ spins. In [Pons et al. 2007, Braungardt et al. 2007] it is detailed how an appropriately chosen set of patterns with eight trapped ions may be used as qubits thus forming a quantum neural network. In particular, single-qubit gates and two-qubit gates by adiabatic passage are worked out that make use of additional effective fields.

\subsection{Mesoscopic systems}

The question if and to what extent quantum mechanical effects play a role in mesoscopic or even macroscopic systems was posed already shortly after quantum theory was developed [Schrödinger 1935]. Except concerning some particular physical phenomena, for example, macroscopic currents in a superconducting material, it is usually assumed that superposition states, interference, and entanglement are not relevant when dealing with entities made up from a large number of individual constituents that, in addition, may interact with a noisy environment at non-zero temperature, for example, biologically relevant molecules. Indications that quantum mechanics indeed plays an important role even for large molecules come from recent experiments where interference with $\mathrm{C}_{70}$ molecules was observed [Hackermuller et al. 2004].

A theoretical study of how dephasing caused by coupling to a noisy environment can actually assist in transferring energy (or information), instead of impeding it, across a network of quantum nodes is presented in [Plenio \& Huelga 2008]. It is shown that, in a simplified model, light-harvesting molecules may take advantage of this process. An experimental 
verification directly with these molecules is difficult at present. However, trapped ions may be used to implement a suitable model to test such a prediction. For a first simple demonstration, appropriately chosen internal levels of a single ion may be used to model the nodes of a network and induced and spontaneous transitions between these levels to mimic energy transport.

Another recent theoretical study demonstrates that entanglement may persist in a model two-spin molecule even when it is exposed to a decohering hot environment, a situation typically encountered for biological molecules [Cai et al. 2008]. The time dependent Hamiltonian modeling the two-spin molecule used for this study is

$$
H_{M}(t)=J(t) \sigma_{x}^{1} \sigma_{x}^{2}+B(t)\left(\sigma_{2}^{1}+\sigma_{z}^{2}\right) .
$$

This molecule is then coupled to a noisy environment modeled by a master equation. After performing a unitary transformation such that $\sigma_{x} \rightarrow \sigma_{z}$ and $\sigma_{z} \rightarrow-\sigma_{x}$ we recognize a timedependent variant of the quantum transverse Ising model 4 which could be realized with an ion spin molecule by using the spin-coupling present in equation 15 and by adding a driving field as in equation 21 .

\section{Quantum Optics Simulating Quantum Optics}

The formal similarities for creation and annihilation operators for photons and phonons on one side, and spin $S=1 / 2$ particles on the other side suggests the equivalence of various and seemingly different experiments within the field of quantum optics. In this notion, a Ramsey measurement might be regarded as simulating a Mach-Zehnder interferometer, since a wavefunction is coherently split and recombined after a independent time-evolution of its constituents. Therefore one general proposition which holds also for other classes of experiments discussed in this paper is maybe of particular importance here: simulation goes both ways. If the Hamiltonian describing a setup in an ion trap, possibly including static and radiation fields, is equivalent to the Hamiltonian of another system, then the ion trap experiment can be thought as a simulation of the other system of interest. But on the other hand it also means, that these other system might reveal some insights about certain aspects and experiments in ion traps, and, depending on the specific technical difficulties when trying to perform it in an ion trap, it might also be imaginable to simulate some aspects of ion trap physics in other systems. As pointed out earlier, however, trapped laser-cooled ions are a flexible, well controlled and well understood system, which can be brought into a variety of regimes, where the physics resembles other systems. Nowadays we are familiar with the fact that both photons but also the internal states of particles can be entangled or combinations of them and it might sound unusual to phrase any of such experiments as a simulation of the other. Nevertheless the creation and detection of entanglement of particles or phonons is subject to different problems and certain aspects of entanglement and its meaning in investigating fundamental questions of quantum mechanics might best be looked at in either approach [Wineland, Monroe, Itano, King, Leibfried, Myatt \& Wood 1998].

\subsection{Theoretical Background}

Already in the early days of quantum information it became clear that quantum computing at a level of factorizing large numbers would be a daunting task (see [Wineland, Monroe, Itano, King, Leibfried, Myatt \& Wood 1998]), though, since then, astonishing progress was made this field. The first articles considering quantum simulations were, to some extent, motivated by the insight that the requirements for quantum simulations (in terms of gate fidelity etc.) are 
less stringent despite their potential to investigate systems that are beyond reach of simulations with classical computers. Despite all progress, this statement still holds and is one motivation for this topical review.

The experiments in quantum optics that are considered in [Wineland, Monroe, Itano, Leibfried, King \& Meekhof 1998, Leibfried et al. 1997] can be simulated by trapped ions interacting with radiation fields that drive Raman transitions between hyperfine levels. A similar description can be obtained when this transitions are directly driven using microwaves (using auxiliary inhomogeneous magnetic fields) [Mintert \& Wunderlich 2001, Mintert \& Wunderlich 2003, Wunderlich 2002, Johanning et al. 2009] or when optical transitions between metastable states are used. For a harmonically bound atom interacting with two travelling wave light fields, both detuned from any real state and with frequency difference $\Delta \omega$ and phase difference $\phi$ the Hamiltonian in the interaction picture can be brought into a form $H_{\epsilon l}$ (after rotating wave approximation and adiabatic elimination of the near resonant excited states and in the Lamb-Dicke limit $\left.\eta^{2}\left\langle\left(a+a^{\dagger}\right)\right\rangle \ll 1\right)$ [Wineland, Monroe, Itano, Leibfried, King \& Meekhof 1998] with integer $l$ indicating changes in the motional state, and $\epsilon \in[0,1]$ indicating changes in the internal state. This Hamiltonians are frequently used in experiments with laser cooled ions; to name a few, $H_{10}$ is called the carrier transition, $H_{12}$ is the second blue sideband, $H_{01}$ is called coherent drive, $H_{02}$ is the squeeze drive. Nesting and concatenation operators of the set $H_{01}, H_{02}, H_{03}, H_{10}, H_{11}, H_{12}, H_{13}$ is sufficient to efficiently generate a wide range of Hamiltonians, as, for example the Hamiltonian for a spin $s=1 / 2$ particle with mass $\mu$ in an arbitrary potential [Leibfried et al. 2002].

These chaining of Hamiltonians could be used for a variety of experiments, as, just to highlight a few, the phonon maser [Wallentowitz et al. 1996] and three phonon down conversion [Wineland, Monroe, Itano, King, Leibfried, Myatt \& Wood 1998] and has been used to create motional cat staes [Monroe, Meekhof, Leibfried, King, Itano \& Wineland 1996], coherent states [Meekhof et al. 1996a, Meekhof et al. 1996b], and to create a Hamiltonian similar to two photon excitation in cavity QED [Leibfried et al. 1997]. An example discussed in the following subsection is an an experimental analogy to a nonlinear two beam (e. g. Mach-Zehnder) interferometer as proposed in [Wineland, Monroe, Itano, King, Leibfried, Myatt \& Wood 1998]. Using ions for the implementations allows for high state preparation and detection efficiency and eliminates the need for data postselection.

All the experiments above used only a single ion and innumerable possibilities come into reach using multiple ions. The first demonstration of the CNOT gate [Cirac \& Zoller 1995] was a step in this direction using, however, a single ion and its motional degree of freedom [Monroe, Meekhof, King, Itano \& Wineland 1995]. This concept can be adapted to entangle different atoms when it is carried out after the state of one ion is mapped onto the centre of mass (CM) mode and afterwards mapped back [Schmidt-Kaler et al. 2003, Leibfried et al. 2003].

\subsection{Experiments}

As a demonstration of a quantum simulation with a single ion, the Hamiltonians $H_{11}, H_{12}, H_{13}$ are used in [Leibfried et al. 2002] to simulate a set of two nonlinear beam splitters forming a nonlinear Mach-Zehnder. A single ${ }^{9} \mathrm{Be}^{+}$ion provides the internal state $|a\rangle$ with

$$
\begin{aligned}
|0\rangle_{a} & =\left|F=1, m_{F}=-1\right\rangle \\
|1\rangle_{a} & =\left|F=2, m_{F}=-2\right\rangle
\end{aligned}
$$


and the motional state $|n\rangle_{b}$ labelling the $n$th vibrational state. The experimental sequence is depicted in 6 and is as follows: the ion is cooled to the ground state of motion $|1\rangle_{a}|0\rangle_{b}$. A $\operatorname{Raman} \pi / 2$ pulse applied on the appropriate sideband is used to create the state proportional to $|1\rangle_{a^{\prime}}|0\rangle_{b^{\prime}}+|0\rangle_{a^{\prime}}|n\rangle_{b^{\prime}}$. This is equivalent to a beamsplitter: analogous to coherently splitting light into two separate directions, the population is coherently split into two states which, in this case, differ in both internal and motional quantum number. Subsequently the potential of the endcap electrodes is changed yielding trap frequency change $\Delta \nu_{z, 1}$ for a time $t$ which introduces a phase shift $n \Delta \nu_{z, 1} t=n \phi$ when the ion is in the $n$th vibrational level. Finally a second $\pi / 2$ pulse on the same sideband is applied which maps the phase difference to the population in the internal states $|0\rangle_{a}$ and $|1\rangle_{a}$ which is measured by Doppler cooling and fluorescence detection. The absolute acquired phases are irrelevant as is the total arm length of a Mach-Zehnder interferometer: longer arms only tend to make it more susceptible to perturbations, however, the signal at the outputs is given by the differential phase shift. The sequence is repeated to improve signal to noise ratio and to extract the sinusoidal time evolution in the different states.

The experiment can be interpreted as nonlinear Mach-Zehnder interferometer. A nonlinear beamsplitter can annihilate a photon in mode $a$ while creating $n$ photons in mode $b$ (or vice versa). The annihilation operator $a$ is replaced in this experiment by the atomic raising operator $\sigma^{+}$between the states $\left|F=2, m_{F}=-2\right\rangle$ and $\left|F=1, m_{F}=-1\right\rangle$. These two operators are not equivalent but they act the same as long as the $\left\{|0\rangle_{a},|1\rangle_{a}\right\}$ subspace is not left, which is fulfilled as long as the input state is $|1\rangle_{a}|0\rangle_{b}$. The optical mode with lowering operator $b$ is replaced by the motion along a selected direction and phonon number states $|n\rangle_{b}$. A $n$th order nonlinear beamsplitter is then given as

$$
B_{n}=\hbar \Omega_{n}\left[a\left(b^{\dagger}\right)^{n}+a^{\dagger}(b)^{n}\right] .
$$

When this operator brackets an operator that causes a phase shift in one arm by $n \phi$, the system resembles a Mach-Zehnder interferometer and the probability of detecting a phonon in output $a^{\prime \prime}$ becomes

$$
P_{a^{\prime \prime}}=\frac{1}{2}[1-\cos (n \phi)]
$$

This experiment requires a sideband transition in the $n$th sideband, which can be very inefficient with increasing $n$ as the sideband Rabi frequencies scale with $\eta^{n}$ and the preparation becomes slow. An faster alternative route exploiting an auxiliary state and first order sidebands is detailed in [Wineland, Monroe, Itano, King, Leibfried, Myatt \& Wood 1998].

An increase in fringe frequency proportional to the order of the nonlinearity is found but the fringe contrast is reduced with increasing $n$, as preparation and manipulation imperfections spoil the fidelity of the states and the detection efficiency is below one for all experiments. The sensitivity of the output signal to phase changes is maximized at the steepest slope of the signal and the signal to noise ratio for the $n=2$ and $n=3$ measurements is found to be below a perfect first order interferometer with unit detection efficiency. A potential improvement by increasing $n$ from 2 to 3 is almost perfectly cancelled by the reduction of fringe contrast in the data presented in [Leibfried et al. 2002].

\section{Transverse standing wave: Bose-Hubbard-physics (and beyond)}

Laser cooling and trapping has been considered [Hänsch \& Schawlow 1975, Wineland \& Dehmelt 1975] and successfully applied (see [Ghosh 1995, Metcalf \& van der Straten 2001], 


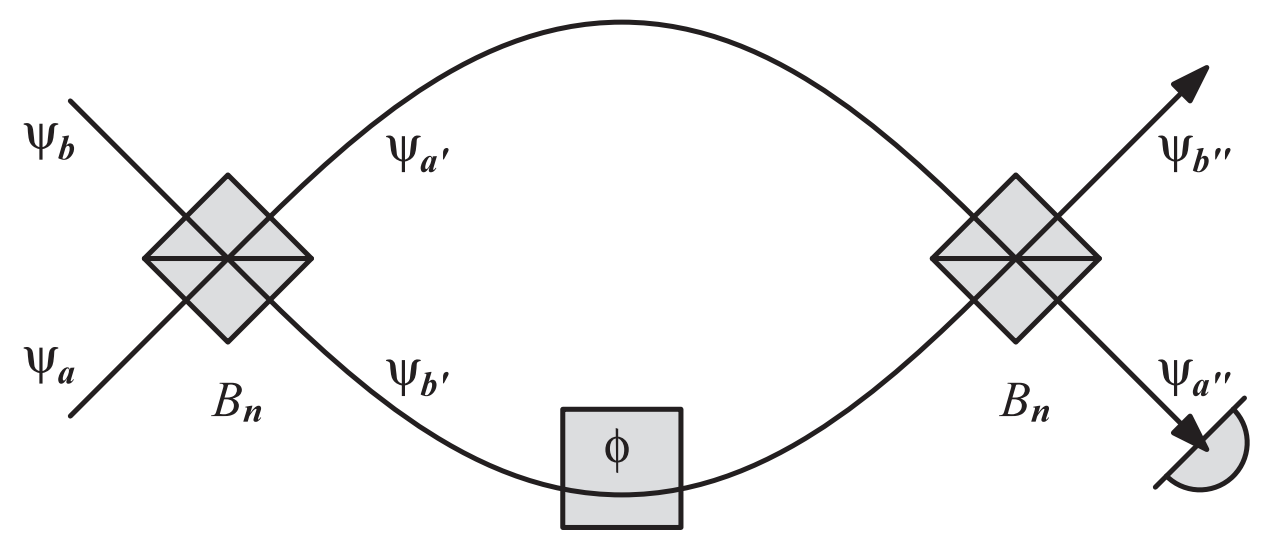

Figure 6. Ion trap Mach-Zehnder interferometer: the manipulation of the wavefunction of a trapped ion can be thought analogous to a set of two nonlinear beamsplitters forming a nonlinear Mach-Zehnder interferometer.

and references therein) to both neutral atoms and ions. Both types of laser cooling experiments are often very much alike in terms of the description of the experiment (trap frequencies and depths, Rabi frequencies etc.) but also in terms of experimental techniques. A recent topic in cold neutral atom physics is the combination of cold trapped atoms and optical lattices which allows to experimentally investigate the Bose Hubbard model [Fisher et al. 1989], which is related to the Hubbard model in solid state physics [Hubbard 1963]. The freedom in changing tunnelling and interaction parameters, the defect free optical lattices and the controlled introduction of disorder [Schulte et al. 2006] allow nice and clean experiments [Greiner et al. 2002, Morsch \& Oberthaler 2006]. Also fermionic atoms have been loaded into traps and lattices and brought to quantum degeneracy. The description of such experiments by the Bardeen-Cooper-Schrieffer (BCS) theory [Bardeen et al. 1957, Giorgini et al. 2008] is known from superconductivity and this analogy encourages the hope that this experiments can help to investigate and understand high $T_{c}$ superconductivity [Hofstetter et al. 2002, Holland et al. 2001].

The large and long range Coulomb force being the dominant force in ion traps has a number of consequences: trap frequencies, trap depths, interaction energies, and particle separations are typically higher by orders of magnitude compared to traps for neutral atoms. Thus Bose-Einstein condensation (and also Bose-Hubbard physics), one long sought holy grail in quantum optics, has therefore been investigated with neutral atoms but not with ions: when cooling neutral atoms at sufficient density, their wave functions can overlap and a macroscopic population of the trap ground state forms [Anderson et al. 1995, Davis, Mewes, Andrews, van Druten, Durfee, Kurn \& Ketterle 1995]. When on the other hand an ion chain is cooled to the lowest vibrational mode [Diedrich et al. 1989], the ion separation is orders of magnitude larger than the spatial extension of the wave function.

But quantum degeneracy and Bose Hubbard physics can still be simulated in ion traps when the ions take the role of lattice sites which can be populated by phonons, more specifically excitations of the vibrational modes transverse to the weak trap axis. The phonons therefore represent the neutral atoms located in one lattice site and phonons can tunnel between sites as do the atoms in optical lattices. Furthermore, as we will see later on, the phonons can be made interact by anharmonicities in trapping potential. This anharmonicities 
can be introduced by the light shift of an off-resonant standing wave. This could be used to demonstrate a Bose-Einstein condensate (BEC) of phonons [Porras \& Cirac 2004b], the superfluid-Mott insulator transition [Deng et al. 2008] and, at higher levels of interaction energy, the Tonks-Girardeau gas [Deng et al. 2008] and even frustrated $X Y$-models [Schmied et al. 2008]. In 1D, BEC at non-zero temperature is only possible when the number of atoms (or here phonons) is finite, and we are in the weak coupling regime, which means, that the average interaction energy is smaller than the expectation value of the kinetic energy. A peculiarity of the effective phonon interaction being dependent on the position of the standing wave is that it can be tuned, even in sign, similar as a the magnetic field can change the scattering length near a Feshbach resonance.

Besides the general appeal to demonstrate Bose-Einstein condensation in yet another system, Bose-Hubbard physics in ion traps offers advantages, and also drawbacks, over present neutral atom experiments: experiments in ion traps do not only offer global control of tunnelling rate and interaction energy (as we will see later), equivalent to ramping the optical lattice depth or applying a magnetic field to obtain a certain scattering length near a Feshbach resonance. Ion traps allow also for local control: irregular lattices correspond to site dependent tunnelling rates and can be obtained in segmented microtraps; the interaction energy can as well be made site dependent by having independent standing wave intensities and phases at the different ion positions - in terms of neutral atoms this would correspond to arbitrary $3 \mathrm{~d}$ Feshbach magnetic fields. Other interesting features include single site control and readout of the state and the potential to extract the density matrix either partially or, even fully (in small systems). On the other hand the experiments discussed here are restricted to bosonic phonons and thus Bose-Hubbard physics and today's ion traps are mostly restricted to one dimension but true two-dimensional systems can be obtained in surface traps [Seidelin et al. 2006].

\subsection{Background}

When we speak of phonons in the context of linear ion traps, we often think in terms of axial normal modes. In this situation momentum is transferred by local interaction with the addressed ion which couples efficiently to the motion of the whole chain due to changing separations and acting Coulomb forces. In this chapter radial modes of the ion motion are considered. Classically a radial displacement of a single ion excites both radial motion of other chain members but also the axial motion of the whole chain. But when the axial ion separations are much larger than radial excursion of the ion motion, the coupling of this motion to all other modes is very inefficient: the motion is almost perfectly perpendicular to the Coulomb interaction between ions all the time and does not change the ion separations to first order. Thus radial phonons can be viewed as being located to one ion but the non vanishing coupling makes the localization non permanent and re-emerges as a finite phonon tunnelling rate.

The Hamiltonian describing the system reads [Deng et al. 2008]

$$
H_{0}=\sum_{i=1}^{N} \frac{p_{i}^{2}}{2 m}+V_{T}+\sum_{i, j=1, i>j}^{N} \frac{1}{4 \pi \epsilon_{0}} \frac{e^{2}}{\left|\vec{R}_{i}-\vec{R}_{j}\right|}
$$

where the trapping potential $V_{T}$ could be simply quadratic like the effective potential created by a simple linear Paul trap, or be array of microtraps achieved by micro structuring [Rowe et al. 2002, Stick et al. 2006]. We will look in more detail into one radial coordinate $x$ and excitations at the trap frequency $\nu_{x, 1}$ in this direction. The trap frequency $\nu_{y, 1}$ can be chosen to be non-degenerate to allow for addressing specific sidebands and to avoid unwanted 
a)

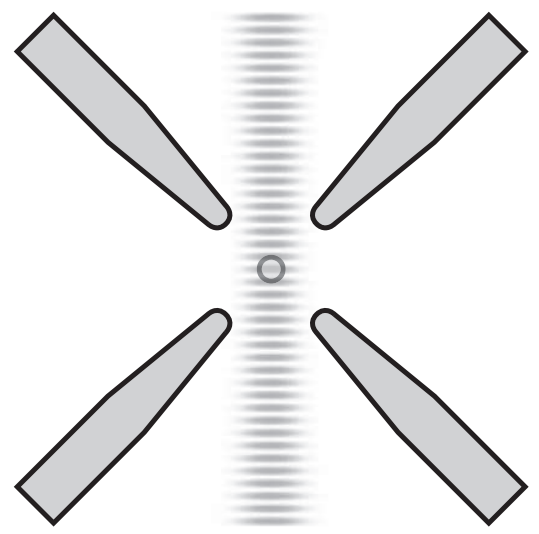

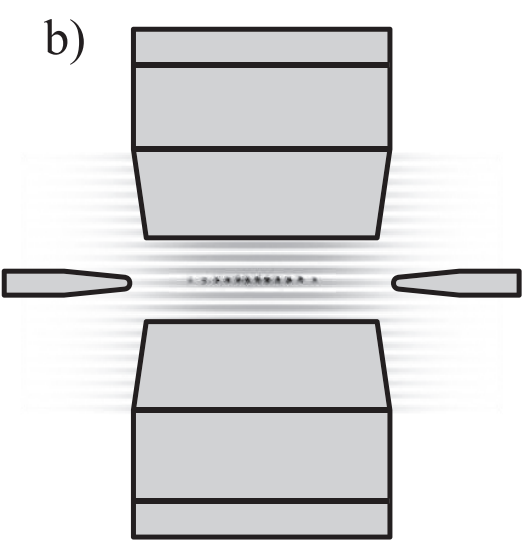

Figure 7. Experimental setup (not to scale) for the simulation of Bose-Hubbard physics. Images a) and b) are sketches of the trap and the applied standing wave potential. The illustrations are not drawn to scale, especially the wavelength of the standing wave is greatly exaggerated for visual clarity. Figure a) shows the trap as seen from a one of the endcap electrodes. Figure b) shows the setup from the side, as seen in most cases from an imaging detector; the weak trap axis is perpendicular to the standing wave.

couplings. The absolute value of the Coulomb force between two particles separated by a large distance $\delta z$ in $z$-direction and displaced only slightly by $\delta x$ in the $x$-direction is

$$
|\vec{F}|=\frac{e^{2}}{4 \pi \epsilon_{0}} \frac{1}{\delta x^{2}+\delta z^{2}} \approx \frac{e^{2}}{4 \pi \epsilon_{0}} \frac{1}{|\delta z|^{2}}
$$

The component in $x$-direction of the Coulomb force is given by

$$
F_{x}=|\vec{F}| \frac{\delta x}{\sqrt{\delta x^{2}+\delta z^{2}}} \approx|\vec{F}| \frac{\delta x}{|\delta z|} .
$$

When the radial separation $\delta x$ is identified with $x_{i}-x_{j}$ and the axial separation $\delta z$ with the unperturbed $z_{i}^{0}-z_{j}^{0}$, the radial potential for small relative displacements becomes

$$
H_{\text {Coulomb }, x} \approx-\frac{1}{2} \frac{e^{2}}{4 \pi \epsilon_{0}} \sum_{i, j=1, i>j}^{N} \frac{\left(x_{i}-x_{j}\right)^{2}}{\left|z_{i}^{0}-z_{j}^{0}\right|^{3}}
$$

Introducing the quantities

$$
\begin{aligned}
t_{i, j} & =\frac{1}{2} \frac{1}{4 \pi \epsilon_{0}} \frac{e^{2}}{m \nu_{x, 1}^{2}} \frac{\hbar \nu_{x, 1}}{\left|z_{i}^{0}-z_{j}^{0}\right|^{3}} \\
\nu_{x, 1}^{(i)} & =\nu_{x, 1}-\sum_{j=1, j \neq i}^{N} t_{i, j}
\end{aligned}
$$

the second quantized form becomes [Deng et al. 2008]

$$
\frac{H_{x 0}}{\hbar}=\sum_{i=1}^{N} \nu_{x, 1}^{(i)} n_{i}+\sum_{i, j=1, i>j}^{N} t_{i, j}\left(a_{i}^{\dagger}+a_{i}\right)\left(a_{j}^{\dagger}+a_{j}\right)
$$

The quantities $t_{i, j}$ are site dependent hopping terms resulting from the non vanishing coupling of the radial motion between all ions in the chain. Coupling of ion motions and 


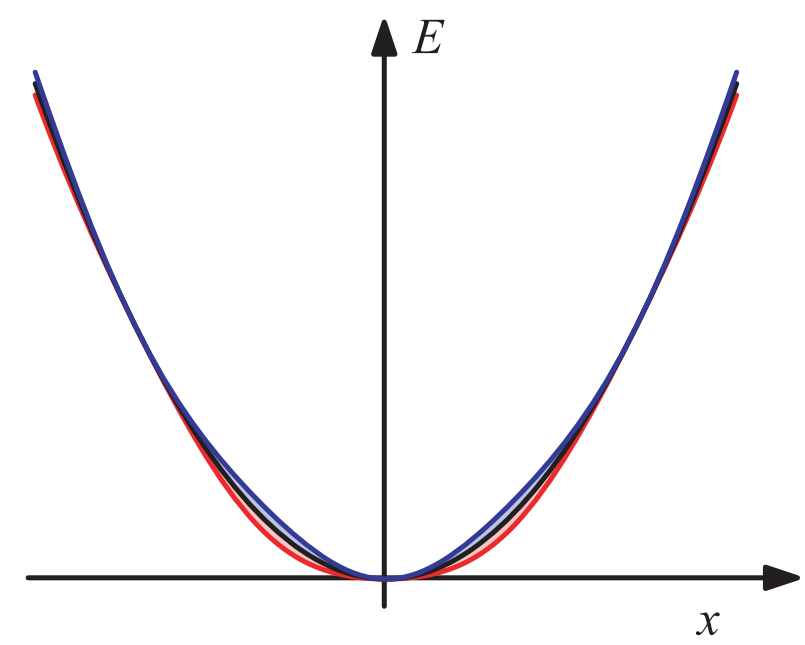

Figure 8. (colour online) The transverse potential as a sum of a parabolic trapping potential plus a far off-resonant standing wave: the black curve shows the unperturbed effective trap potential, while the red is the total effective potential with a standing wave having a maximum at the ion position, resulting in a effective repulsive interaction of phonons. The blue shows the total effective potential with a standing wave having a node at the ion position, resulting in a effective attractive interaction of phonons.

therefore tunneling of transverse phonons is the stronger the closer the ions are. The nextneighbour tunnelling is therefore the strongest but its slowly decaying behaviour with lattice site difference can already be seen from equation (39), leading to long range tunnelling in stark contrast to pure next-neighbour tunnelling, as found in neutral atom experiments. The Coulomb repulsion from adjacent ions has a maximum for $\delta x=0$ and counteracts the radial trapping potential, and the $\nu_{x, 1}^{(i)}$ are the reduced radial trapping frequencies. These changes in the radial trapping frequencies and therefore the reductions in the phonon energies are the strongest for small ion separations and thus lead to an axial phonon confinement towards the centre of the trap [Deng et al. 2008]. This confinement becomes the stronger, the more inhomogeneous the ion separations are. Therefore this effect is more pronounced in a linear ion trap compared to the isospaced array of microtraps (with almost constant ion separations) and becomes more evident with increasing ion number.

Due to the large separation of ions and the small transverse oscillation amplitudes, the effect of the Coulomb interaction to the radial potential is just a change in the trapping frequency and the anharmonicities, that should in principle be already present, have been neglected so far. A purely harmonic trapping potential results in equally spaced oscillator levels, meaning that the energy of having two phonons located on different sites or both at the same site gives the same total energy. Interacting phonons means that the energy of having two phonons at the same site is the sum of the the two single phonon energies, altered by an interaction energy, and the oscillator levels are no longer equidistant. A nifty idea to mimic this interaction is to introduce an anharmonicity to the trapping potential and thus to break the regular spacing of the trap levels. This can in principle be done by any non harmonic potential as, for example a sinusoidal potential as known from optical lattices generated by an 
off-resonant standing wave with wave vector $k_{\mathrm{SW}}$ [Deng et al. 2008]:

$$
H_{\mathrm{SW}}=F \sum_{i=1}^{N} \cos ^{2}\left(k_{\mathrm{SW}} x_{i}+\frac{\pi}{2} \delta\right)
$$

where $F$ is the peak AC Stark energy shift. For small anharmonicities the Hamiltonian $H_{x 0}+H_{S W}$ can be expanded in the Lamb-Dicke-parameter $\eta_{x, \text { SW }}$ with

$$
\eta_{x, \mathrm{SW}}=\sqrt{\hbar^{2} k_{\mathrm{SW}}^{2} / 2 m \hbar \nu_{x, 1}}
$$

and is sufficiently approximated when truncated after second order terms and the Hamiltonian can be brought into the well known form of the Bose-Hubbard model [Deng et al. 2008, Fisher et al. 1989]:

$$
\begin{aligned}
H_{x}^{B H M}= & \sum_{i, j=1, i>j}^{N} \hbar t_{i, j}\left(a_{i}^{\dagger} a_{j}+\text { H.c. }\right) \\
& +\sum_{i=1}^{N} \hbar\left(\nu_{x, 1}+\nu_{x, 1}^{(i)}\right) a_{i}^{\dagger} a_{i} \\
& +U \sum_{i=1}^{N} a_{i}^{\dagger 2} a_{i}^{2}
\end{aligned}
$$

In this approximation of small anharmonicities, the total radial phonon number is conserved and coupling to axial modes is neglected. The third term in equation 44 has a contribution proportional to the phonon number squared which is responsible for the interaction. Its strength is scaled by [Deng et al. 2008]

$$
U=2(-1)^{\delta} F \eta_{x, \mathrm{SW}}^{2}
$$

The interaction depends on the strength and the relative position $\delta$ of the standing wave and can even change its sign (see equations 42 and 45): it is maximum positive/repulsive for ions at the maxima of the standing wave $(\delta=0)$ and maximum negative/attractive for ions at the minima of the standing wave $(\delta=1)$. This can be seen in figure 8 and 9 the small sinusoidal alteration from the standing wave makes the tip of the parabola slightly pointier for a minimum of the standing wave or chamfered for a maximum of the standing wave which results in a shift of the trapping frequency. The interesting consequence, however, are the differential shifts of the oscillator levels which are depicted in figure 9. the fourth order contribution from the extremum of the sinusoidal potential has always opposite sign of the quadratic contribution. Thus it is curved upwards for a maximum of the standing wave (meaning that the quadratic term is curved downwards) and shifts higher levels up, mimicking a repulsive interaction. A minimum of the standing wave has an upwards curved quadratic contribution; the fourth order is curved downwards, making the trap softer when walking away from the centre and reduces the energy of higher trap levels imitating an attractive interaction.

The Bose-Hubbard model is well known in neutral atom physics [Fisher et al. 1989]. When the total phonon number $N_{\mathrm{ph}}$ is an integer multiple of the ion number $N_{\text {ion }}$ the model predicts a transition from the superfluid state (dominated by tunnelling) to a Mott-insulator state (negligible tunnelling) with a constant phonon number per lattice site. This transition can be traversed by changing the ratio of tunnelling frequency and repulsive interaction energy (that is, changing trap frequency or standing wave intensity). For incommensurate phonon numbers that are no integer multiple of the ion number, a finite number of stages with constant phonon occupation can be anticipated, with the highest phonon populations at the bottom of the phonon confining potential, that is, at the trap centre. In neutral atom physics, 


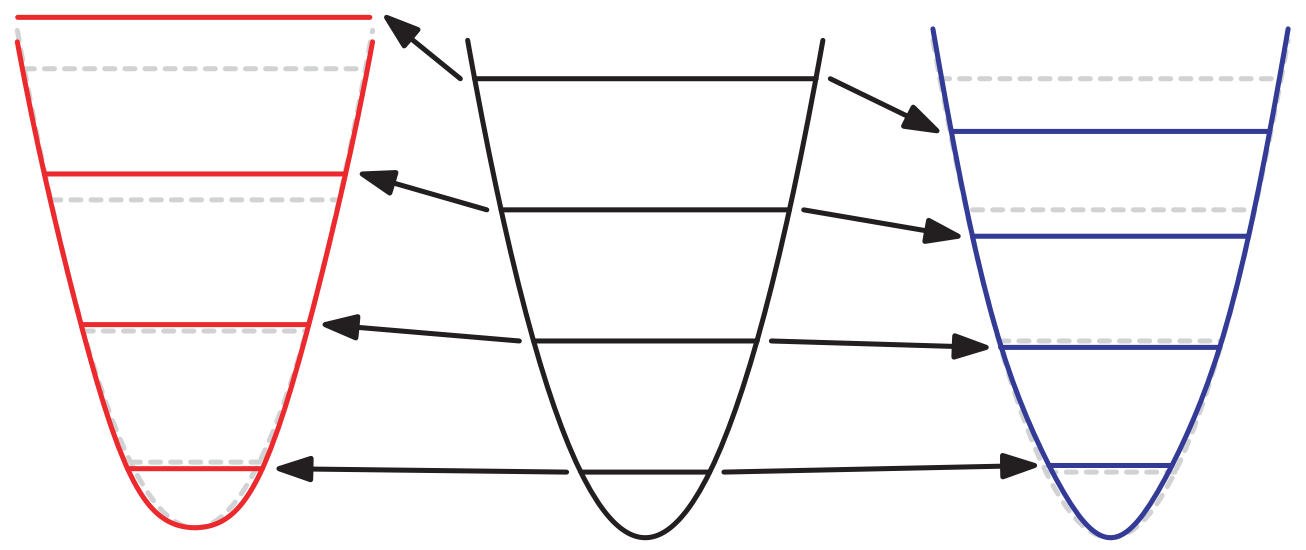

Figure 9. (colour online) Shifts of the harmonic oscillator levels due to the standing wave (not to scale). The centre image shows an undistorted harmonic trap with equally spaced energy levels. When a standing wave along $x$ is added, such that a maximum is located at the trap centre (left hand side), the base level is lowered due to the quadratic part in the standing wave. In addition, the higher levels are raised due to the quartic part of the dipole potential. The levels spacing is no longer equidistant and mimics a repulsive interaction of the phonons. For an additional standing wave with a minimum at the trap centre (right hand side), the lowest level is raised due to the upward curvature of the dipole potential. The level spacing is reduced and also non equal and pretends a attractive interaction of the phonons. The grey dashed lines in the outer graphs again indicate unperturbed potential and energy levels.

the superfluid-Mott insulator transition has been observed in beautiful experiments [Greiner et al. 2002].

The framework of Luttinger liquid theory [Luttinger 1963, Voit 1995] predicts algebraic decay of correlations between the number of phonons for the superfluid phase when phonon tunnelling beyond next-neighbours is neglected [Deng et al. 2008]

$$
C_{i, j}^{n n}=\left\langle n_{i} n_{j}\right\rangle-\left\langle n_{i}\right\rangle\left\langle n_{j}\right\rangle \propto|i-j|^{-2}
$$

and correlations that are nondiagonal in the phonon number decay as

$$
C_{i, j}^{a a}=\frac{\left\langle a_{i}^{\dagger} a_{j}\right\rangle}{\sqrt{\left\langle n_{i}\right\rangle\left\langle n_{j}\right\rangle}} \propto|i-j|^{-\alpha}
$$

with $\alpha \propto \sqrt{U / t n_{0}}$ given by the ratio of tunnelling and interaction at the central phonon density $n_{0}$. In the Mott phase, however, both correlations decay exponentially with distance:

$$
C_{i, j}^{a a, n n} \propto e^{-|i-j| / \xi}
$$

with the correlation length $\xi$.

Phonon densities and fluctuations from numerical calculation using the density matrix renormalization group method (DMRG) [White 1993] were presented in [Deng et al. 2008] for linear traps and an array of microtraps. The calculations reproduced the superfluid Mottinsulator transition starting from the sides of the chain (due to larger ion separations, the tunnelling rate is lower at the ends). Especially for an array of microtraps the Mott phase features an almost flat mean phonon number per lattice site along the chain for commensurate ion and phonon number and the fluctuations are also flat and strongly reduced. For linear traps the inhomogeneous ion separations modulate phonon numbers and fluctuations which 
are both more peaked at the chain centre. The correlations in the superfluid phase are found to be in agreement with (4647)) for short (linear trap) and intermediate (microtrap array) distances and signatures of finite size effects and inhomogeneous ion separations can be seen and are found to be more important in linear traps. In addition, long range correlations in Mott phase are found that can be attributed to the long range hopping terms in equation (40). For large repulsive interactions and incommensurate filling the system was found in [Deng et al. 2008] to form a Tonks-Girardeau-gas [Girardeau 1960, Lieb \& Liniger 1963] which has been recently observed in neutral atoms [Paredes et al. 2004, Tolra et al. 2004]. Due to their repulsion, the phonons cannot get past each other and attain the hard core bosons limit and tunnelling is suppressed. The atoms/phonons behave neither purely bosonic nor fermionic, because they can occupy the same momentum state but not the same position in space. Mathematically, there is an exact one-to-one mapping between impenetrable bosons and non interacting fermions for a one-dimensional system. Numerical studies in [Deng et al. 2008] for an array of microtraps and half filling $\left(N_{\mathrm{ph}}=1 / 2 N_{\text {ion }}\right)$ show a constant phonon number distribution together with algebraically decaying correlations. The exponent of the decay deviates from the value $1 / 2$ which is expected for a Tonks gas with nearest neighbour interaction only. This is explained by mapping the BHM to a $X Y$ model with antiferromagnetic dipolar interactions. [Deng et al. 2008] also study attractive systems and use site dependent interactions to construct systems with highly degenerate ground states described by an $X Y$ model.

\subsection{Experiments}

For a string of 50 ions with a minimum axial separation of $5 \mu \mathrm{m}$ and a radial trapping frequency $\nu_{x, 1}$ about 70 times higher than the axial trapping frequency $\nu_{z, 1}$ the tunnelling rates $t_{i j}$ were found to be comparable to the axial trapping frequency. For $F=\hbar \nu_{x, 1}$ which is realistic with commercially available laser systems, the axial frequency is changed by a few ten percent and the system remains in the phonon number conserving regime and the phonon interaction energy $U$ is approximately twice the tunnelling rate [Deng et al. 2008].

The interesting quantities are the number of atoms at each lattice site, corresponding to the number of transverse phonons at each ion, their fluctuations and their correlations. Phonon occupation can not be measured directly but can be mapped on the internal state and the ions' resonance fluorescence is recorded. The mapping is accomplished by sidebandtransitions with carrier Rabi frequency $\Omega$ - note that a sideband doesn't refer to the axial mode as usual but to the radial mode along the $x$ axis here. Since the strength of the sideband transition depends on the expectation value of the phonon number, the transition matrix element, and therefore the vibrational state can be extracted from repetitive measurements [Meekhof et al. 1996a, Meekhof et al. 1996b, Leibfried et al. 1996]. If the ion is not in a Fock state, each phonon population contributes to a $\sin ^{2}(\sqrt{n} \Omega t)$ term in the temporal evolution, which is accordingly given by

$$
P_{\uparrow}=\sum_{n} P(n) \sin ^{2}(\sqrt{n} \Omega t)
$$

with $P(n)$ being the probability of having $n$ phonons. Different frequency components in the temporal evolution can be extracted from Fourier analysis to yield the $P_{n}$ [Meekhof et al. 1996a, Meekhof et al. 1996b, Leibfried et al. 1996] and reveal directly and transition from superfluid to Mott insulator state: For a Mott insulator, all ions are in the same vibrational state and therefore have the same transition matrix element (assuming the Number of phonons being an integer multiple of the ion number). Exciting sideband transitions on all ions 
simultaneously brings all of them in the same internal state, so for appropriately chosen ( $\pi$ pulse) times the whole chain will switch from dark to bright. For a superfluid, however, the phonon expectation value is not a constant along the chain, and there is no universal $\pi$ pulse and the ions will never undergo common oscillations from the dark to the bright state when varying the interaction time. If desired, the whole density matrix can be deduced from quantum state tomography which, of course, becomes tedious or, for all practical purposes, even impossible for long chains.

4.2.1. Superfluid-Mott insulator transition and creation of a superfluid phonon state by adiabatic evolution This experiment is similar to the superfluid-Mott insulator transition by [Greiner et al. 2002] obtained by ramping the optical lattice depth. A clear demonstration, as proposed in [Porras \& Cirac 2004b] would start with an ion chain cooled to a state with zero radial phonons, the "radial ground state". The optical lattice is ramped up to a high intensity $U \gg t_{i j}$ such that the ions sit on a node of the standing wave and the phonons experience a repulsive interaction. A defined number of phonons is introduced by sideband transitions, such that the total phonon number is an integer multiple of the ion number (for example by a $\pi$ pulse on the blue sideband). If now the phonon number is mapped back to the internal state by sideband transitions, a collective switching from the internal bright to the non scattering state should be observed, that is, the time for a $\pi$ pulse is identical throughout the whole chain). Alternatively, the interaction $U$ is adiabatically reduced down to a final strength $U_{f}$ (the standing wave intensity is lowered), such that the system remains in the ground state. At a critical value $U_{f} \approx t_{i j}$ the system undergoes a transition to a phonon superfluid. When measuring phonon occupation numbers by mapping them to internal states by sideband transitions, the phonon numbers can be expected to be inhomogeneous, due to the phonon trapping potential and non negligible tunnelling.

4.2.2. Bose-Einstein-condensation by evaporative laser cooling Evaporative laser cooling for phonons in ion traps sounds like an oxymoron because in neutral atom physics evaporative cooling terms a process free of laser scattering, where only the highest energy atoms can leave the trap [Davis, Mewes, Joffe, Andrews \& Ketterle 1995] and take more than the average thermal energy with them which, provided sufficient fast thermalization) results in a cooler cloud. The analogy is, that in the ion trap with an axial phonon confining potential high phonon occupations (labelled high energy phonons in [Porras \& Cirac 2004b]) can more easily tunnel to the ends of the chain, analogous to hot atoms, and when laser cooling is applied to these ends exclusively, the phonons are removed or evaporated.

A demonstration would start with a Doppler cooled ion chain with a given number of phonons per site. Applying laser cooling at the ends of the ion chain removes or evaporates high energy phonons (or phonon occupation numbers) from the top of the phonon confining potential. A small interaction $U \ll t_{i j}$ allows for a phonon-phonon interaction and therefore for thermalization, equivalent to the need of a non vanishing scattering length for thermalization of neutral atom clouds and finally Bose-Einstein condensation of phonons can be observed. Since this is a 1D system, BEC cannot take place in the thermodynamic limit $N \rightarrow \infty$ at finite temperature, but for a given in number there will be a non-zero transition temperature. In contrast to the Mott insulator (phonon Fock state), the signature of this phase transition is more difficult to extract. One can deduce phonon occupation distribution from series of fluorescence images after sideband transitions of varying time. Evaporative laser cooling for phonons should reveal an abrupt transition from a thermal phonon occupation distribution to the BEC distribution, which corresponds to a distinction of a Gaussian and a 
Thomas-Fermi momentum distribution for neutral atoms.

It could be interesting to modify the axial trapping potential in microtraps such that the equilibrium positions are really equidistant and the phonon trapping potential, probably corresponding to unconfined phonons and a demonstration Bose-Einstein-condensation of free particles, similar to experiments by [Meyrath et al. 2005].

\subsection{Frustrated XY models}

For large anharmonicities, strong repulsion, and low filling factor $N_{\mathrm{ph}} \ll N_{\text {ion }}$ the occupation by two phonons becomes unlikely and the system attains the hardcore Boson limit. The phonons can be mapped to $S=\frac{1}{2}$ spins via the Holstein-Primakoff transformation as $\left(a_{\alpha}^{\dagger} \rightarrow S_{\alpha}^{+}, a_{\alpha} \rightarrow S_{\alpha}^{-}\right.$and $n_{\alpha} \rightarrow S_{\alpha}^{z}+\frac{1}{2}$ ). The resulting Hamiltonian is an XY model with dipolar interactions and a site dependent potential [Schmied et al. 2008]:

$$
H_{S}=2 \sum_{\langle\alpha, \beta\rangle} t_{\alpha, \beta}\left(S_{\alpha}^{x} S_{\beta}^{x}+S_{\alpha}^{y} S_{\beta}^{y}\right)+\sum_{\alpha} V_{\alpha} S_{\alpha}^{z}
$$

Hamiltonians like theses are interesting because they allow to investigate frustrated XY models in one and two dimensions. Frustration is a phenomenon relevant in condensed matter physics and describes the situation where, due to the lattice geometry or competing interactions, the interaction energies cannot be simultaneously minimized which can result to highly non-degenerate ground states with non-zero entropy, even at zero temperature. Such frustrated XY models can now be investigated in 1D and 2D system, that is, in linear chains and planar zigzag structures in linear ion traps and planar structures in surface traps.

The 1D case leads to the $S=\frac{1}{2} \mathrm{XY}$ antiferromagnet with dipolar interactions, which becomes exactly solvable if only next neighbour interactions are considered. By weakening the transverse confinement along one direction the linear chain is no longer the energetically favoured pattern and the ion arrange themselves in a planar zigzag ladder which can be viewed as two linear chains relatively displaced to each other. By variation of the radial trapping potential, the properties of this two-dimensional structure, namely the zigzag amplitude and thus the ratio between the dominant inter $\left(t_{1}\right)$ and intra-chain $\left(t_{2}\right)$ coupling can be tuned. The zigzag amplitude $\xi$, measured in units of the inter ion spacing, is limited by the fact that for a too weak transverse confinement the ion chain spontaneously flips into helical order (around $\xi \approx 0.965$ ). This in turn limits the accessible range of coupling ratios when phonons perpendicular to the zigzag plane are considered. But when exploiting phonons in the ladder plane any $\left|t_{2} / t_{1}\right|>1 / 8$ ratio can be accessed. For low zigzag amplitudes and intrachain couplings, the ladder is found to exhibit long range antiferromagnetic Néel order and undergoes a transition at $\xi \approx 0.461$ to spiral order which shows both spontaneous magnetic order and chiral order.

The chirality can be defined as

$$
\kappa_{i}=4\left(S_{i}^{x} S_{i+1}^{y}-S_{i}^{y} S_{i+1}^{x}\right)
$$

and the chiral order parameter is the averaged chiral correlation over all ion-pairs in the ladder:

$$
\begin{aligned}
O_{\kappa}^{\Delta} & =\frac{1}{L-1-|\Delta|} \sum_{i}\left\langle\kappa_{i} \kappa_{i+\Delta}\right\rangle \\
O_{\kappa} & =\frac{1}{2 L-3} \sum_{\Delta=-(L-2)}^{L-2} O_{\kappa}^{\Delta}
\end{aligned}
$$

When envisioning an experiment measuring this chiral order, we first have to remember that the spins in 51 are not real spins but phonons mapped back via the Holstein-Primakoff 
transformation and that the probability of having double phonon occupation is negligible. The expectation values for the phonon occupation are thus given by the probability of having single phonon occupation. To measure chirality and the chiral order parameter, we have to measure phonons in both transverse modes for each ion and their correlations by sideband transitions. When the measurement is repeated for different radial confinements and thus coupling ratios the transition from Néel to sprial order can be observed. Another interesting feature to look at is a yet not understood "reorientation transition" transition around $\xi \approx 0.8$ found in numerical calculations of up to 20 ions [Schmied et al. 2008] which gets more pronounced for larger ion numbers. Furthermore, the convergence of the numerical algorithm used in [Schmied et al. 2008] shows poor convergence for equal inter and intra chain couplings and thus a measurement of the phonon correlation function in this regime could be interesting.

Even more variations are possible in extended planar lattices, as, for example triangular lattices as considered in [Schmied et al. 2008]. Rotating planar triangular Wigner crystals have been observed in Penning traps [Mitchell et al. 1998] and again they can be viewed as displaced linear chains such that it is possible to define a ratio of inter and intra chain coupling. A variety of phases has been found when the orientation of the a co-rotating standing wave, and therefore vibrational motion is changed relative to the lattice normal [Schmied et al. 2008]. Another approach to implement such lattices is by means of surface traps [Chiaverini \& Lybarger 2008, Seidelin et al. 2006]. The equilibrium ion positions and therefore the ratio of inter and intra chain couplings is given by the effective potential generated by the trap. A variation of the ratio of relevant couplings could be investigated either in a series of surface traps, each designed for a particular coupling ratio, or by electrode geometries which allow for a anisotropic deformation of the triangular lattice. It should be pointed out that such planar traps would in principle allow to implement any desired lattice geometry.

\section{Axial standing wave: The Frenkel-Kontorova-Ion-Model}

The Frenkel-Kontorova-Model (FKM) is a model describing a linear chain of particles with harmonic next neighbour interactions which is exposed to a sinusoidal potential [Frenkel \& Kontorova 1938]. The system of interest might remind the reader of the Hubbard- or Bose-Hubbard-model as discussed in section 4.1, however, the Frenkel-Kontorova-Model is simpler, being classical and one-dimensional, and, on the other hand, focusses more on classical nonlinear dynamics and chaos, and questions of integrability and the persistence of quasi-periodic motion [Braun \& Kivshar 1998]. Despite this simplicity the FKM is able to describe a variety of physical phenomena as crystal dislocations, commensurateincommensurate phase transitions, epitaxial monolayers on a crystal surface, magnetic chains, and fluxon dynamics in Josephson junctions (see [Braun \& Kivshar 2004], and references therein). The FKM is one of the first examples in solid state physics where a one dimensional chain can be used to model an extended two dimensional defect in a bulk [Braun \& Kivshar 1998].

\subsection{Formalism and numerical calculations}

In the basic model the interactions are harmonic and restricted to next-neighbours and the external potential is sinusoidal (see figure 10). Without this external potential the dynamics of the chain can be understood in terms of normal modes and is quasi-periodic. When the potential is switched on but the perturbation is small, that is, below the critical perturbation strength, the system is in the so called sliding phase and the chain can oscillate in the lattice 


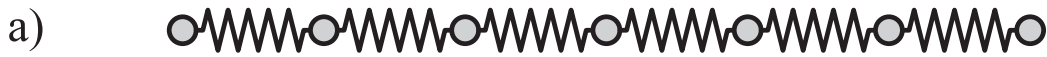

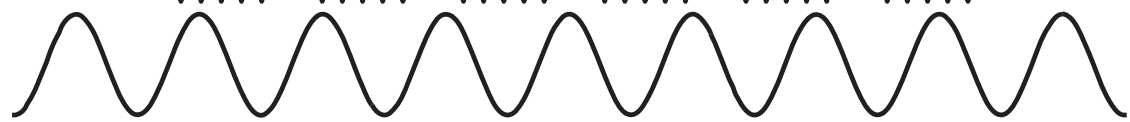

b)

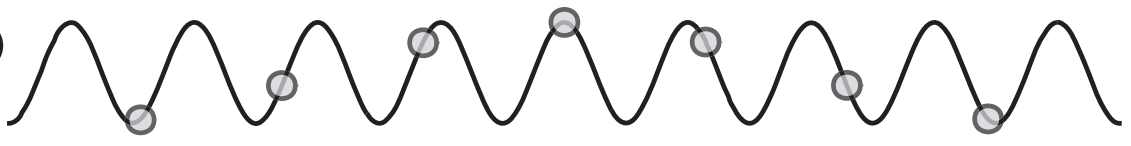

c)

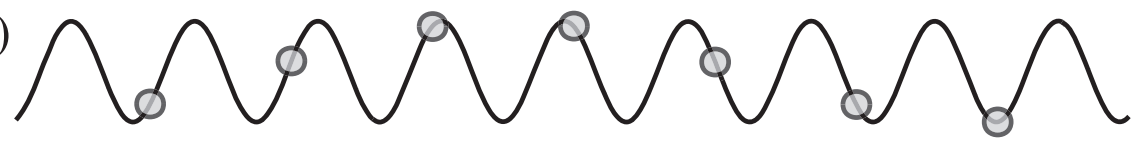

Figure 10. a) basic Frenkel Kontorova model: a one dimensional chain of atoms with next neighbour interactions is exposed to a sinusoidal potential; figures b) and c) show possible spatial configurations: the upper configuration b) is unstable - the total energy, which is a function of the coordinates of all particles has a saddle point, whereas configuration c), obtained only by a minuscule sideways shift is stable, corresponding to a minimum of the total energy (after [Braun \& Kivshar 1998])

and the ion positions follow a Kolmogorov-Arnol'd-Moser curve [Braun \& Kivshar 2004]. For higher perturbations, the system undergoes an Aubry analyticity breaking transition [Aubry \& Le Daeron 1983] and the ion chain starts to be pinned by the lattice and the positions form a devils staircase corresponding to a fractal Cantor set [García-Mata et al. 2007]. The ground state is unique for all perturbations, but in the pinned phase, a large number of states exists very close to the ground state like in a fractal spin glass [Zhirov et al. 2002].

Various extensions and modifications of the basic Frenkel-Kontorova model have been investigated, for example arbitrary on site potentials and anharmonic interactions between the particles [Braun \& Kivshar 1998]. In the following we will focus on the Frenkel-KontorovaIon-Model (FKIM) which is a classical picture for a laser-cooled chain of ions trapped in a linear trap exposed to a standing wave [García-Mata et al. 2007]. For a sample of ions in a linear trap with a much stronger radial than axial confinement, the ions arrange themselves in a linear chain. When the radial motion is frozen due to laser cooling, the chain can be described in good approximation with a one dimensional Hamiltonian. Then the interaction of the particles is given by the long range Coulomb force and the external potential is a sum of the effective sinusoidal potential from the standing wave and the effective harmonic axial trapping potential. The Hamiltonian is given by

$$
\begin{aligned}
H=\sum_{i=1}^{N} & \left(\frac{p_{z, i}^{2}}{2 m}+\frac{m \nu_{z, 1}^{2}}{2} z_{i}^{2}-K \cos \left(2 \pi z_{i} / d\right)\right) \\
& +\sum_{i>j} \frac{e^{2}}{4 \pi \epsilon_{0}} \frac{1}{\left|z_{i}-z_{j}\right|} .
\end{aligned}
$$

The Hamiltonian above corresponds therefore to the general ion trap Hamiltonian given in equation (36) where only the part in $z$-direction is considered and an sinusoidal potential of strength $K$ and periodicity $d$ is added. A basic FKM Hamiltonian can be obtained by neglecting restricting the interaction sum to next neighbours and expanding the sum of interaction and trapping potential in the deviations from the equilibrium positions up to second 


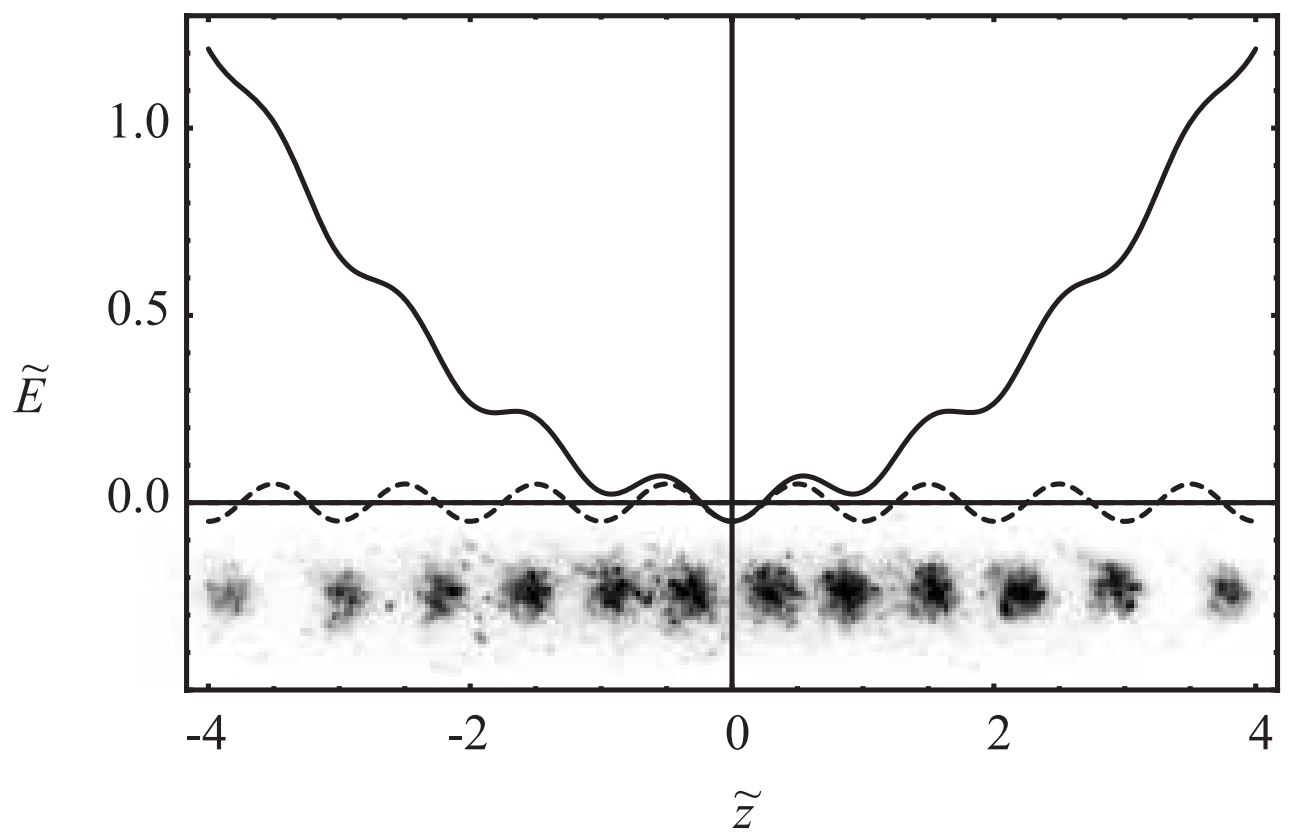

Figure 11. Frenkel-Kontorova ion model: The potential curvature, the sine ripple amplitude and lattice constant are plotted for values corresponding to the Aubry analyticity breaking transition at golden mean density for a chain of 12 ions with a centre separation of $5 \mu \mathrm{m}$. A CCD image of a string of $12^{172} \mathrm{Yb}^{+}$ions is scaled to match the conditions for the potential plot and indicates the unperturbed ion positions.

order. In [García-Mata et al. 2007] the authors investigate the dimensionless form of the FKIM-Hamiltonian above which reads

$$
H=\sum_{i=1}^{N}\left(\frac{P_{i}^{2}}{2}+\frac{\tilde{\nu}^{2}}{2} \tilde{z}_{i}^{2}-\tilde{K} \cos \tilde{z}_{i}\right)+\sum_{i>j} \frac{1}{\left|\tilde{z}_{i}-\tilde{z}_{j}\right|} .
$$

The ion positions $\tilde{z}$ are given in units of the reduced lattice constant $\tilde{d}=d / 2 \pi$ and the energy $\tilde{E}$ as well as the strength of the sinusoidal potential $\tilde{K}$ are measured in units of $e^{2} / 4 \pi \epsilon_{0} \tilde{d}$. The modified angular frequency is obtained as $\tilde{\nu}^{2}=4 \pi \epsilon_{0} m \nu_{z, 1}^{2} \tilde{d}^{3} / e^{2}$. In the quantum case the momenta are given by $P_{i}=-i \hbar_{\mathrm{eff}} \partial_{x_{i}}$ with an effective Planck constant $\hbar_{\text {eff }}$ given as

$$
\hbar_{\mathrm{eff}}=\hbar /(e \sqrt{m d})
$$

which depends on the lattice constant which allows to scale and investigate quantum effects as we will see later.

First the authors compare numerical solutions to the classical ground state problem in the FKIM to solutions of the FKM with only next neighbour interactions. The numerical studies are performed for up to a few hundreds of ions at the golden mean density (at the center of the ion chain), where the average occupation of a lattice site is $(\sqrt{5}+1) / 2$ and results in the golden Kolmogorov-Arnol'd-Moser (KAM) curve for the ion positions commonly used for studies of the Aubry transition [Aubry \& Le Daeron 1983, Braun \& Kivshar 2004]. The FKM leads to dynamical recursive maps for the calculation of equilibrium ion positions which are found to describe the centre $1 / 3$ part of the ion chain appropriately. By comparison of the solutions 
for FKM and FKIM equilibrium positions, the influence of long range interactions and the trapping potential can be mapped out and is found to be negligible in the centre region. Here, the ion density is highest and almost constant and allows for screening of the trap potential by nearby ions, similar to the Debye radius in plasma physics. In the outer regions visible deviations in the equilibrium positions are found, as the ion density is lower at the ends of the chain.

As for the FKM, for small depths of the sinusoidal potential $(\tilde{K}<0.05)$, the chain is in a sliding phase. The hull function, which compares the unperturbed equilibrium positions to the perturbed ones modulo $2 \pi$ is continuous and the phonon mode spectrum has a sound like form. At a critical strength of the standing wave $\left(K_{c} \approx 0.05\right)$, the system undergoes an Aubry analyticity breaking transition [Aubry \& Le Daeron 1983]. Above the critical lattice strength, the ion chain is in a pinned phase, their positions are described by a devils staircase, which is a fractal cantor set [Peitgen et al. 2004], and the phonon spectrum shows a gap. Another similarity to the FKM is that in the pinned phase, the FKIM has properties of a spin glass, and features an enormous number of stable equilibrium configurations energetically close to the ground state [Zhirov et al. 2002], despite the ground state being unique.

So far, all considerations have been purely classical. Quantum effects were in investigated in [Borgonovi et al. 1989] and the quasi-degenerate configurations close to the ground state are found to become important and tunnelling between them leads to nontrivial instanton excitations [García-Mata et al. 2007]. The quantum case is studied in [García-Mata et al. 2007] using the Quantum-Monte-Carlo (QMC) approach [Grotendorst et al. 2002, Nightingale \& Umrigar 1998]. To see this structural changes, the authors look at the power spectrum of the position distribution (in the classical case, the $z_{n}$ are the ion positions):

$$
F(k)=\frac{\left\langle\left|\sum_{n} \exp \left(i k z_{n}(\tau)\right)\right|^{2}\right\rangle}{\delta N}
$$

From these numerical solutions a phase transitions from a pinned instanton glass to a sliding phonon regime is observed, depending on the value of the effective Planck constant $\hbar_{\text {eff }}$ [García-Mata et al. 2007] which can be varied by changing the lattice constant and thus the influence of tunnelling, and the transition taking place at $\hbar_{\mathrm{eff}} \approx 1$. For low values of $\hbar_{\text {eff }}$ the formfactor shows discrete resonances which are equidistant in the sliding phase, and and integer $k$ showing that the ions are pinned by the optical lattice. The pinned phase also shows a quantum transition when $\hbar_{\mathrm{eff}}$ is increased: for low values $\hbar_{\mathrm{eff}}$, the pinned phase is not destroyed and discrete resonances are still found. For larger values of $\hbar$ the formfactor bacomes continuous and the transition occurs at a value $\hbar_{\mathrm{eff}} \approx 1$.

\subsection{FKIM Experiments}

Experiments for investigating Frenkel-Kontorova-physics require a sinusoidal potential along a one-dimensional ion chain. This can be implemented by a far off-resonant standing wave along the weak trap axis as indicated in figure 12. Being far off resonant means that changes of the internal state due to this lasers is unlikely within the duration of the xperiment. Following [García-Mata et al. 2007] the ion density at the centre should be the golden mean density, that is, the lattice periodicity should be by a factor $(\sqrt{5}+1) / 2$ larger than the ion separation at the middle of the chain. This can easily be obtained even at a fixed wavelength changing the angle between the intersecting beams that form the dipole potential or, alternatively, by changing the trap frequency. To obtain the golden mean density for ions separated $5 \mu \mathrm{m}$, the lattice 
a)
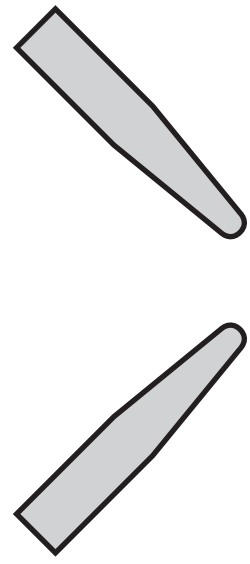

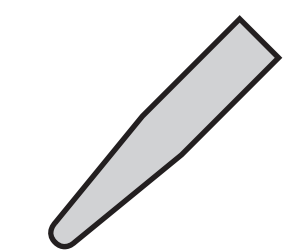

(

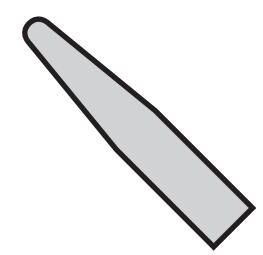

b)

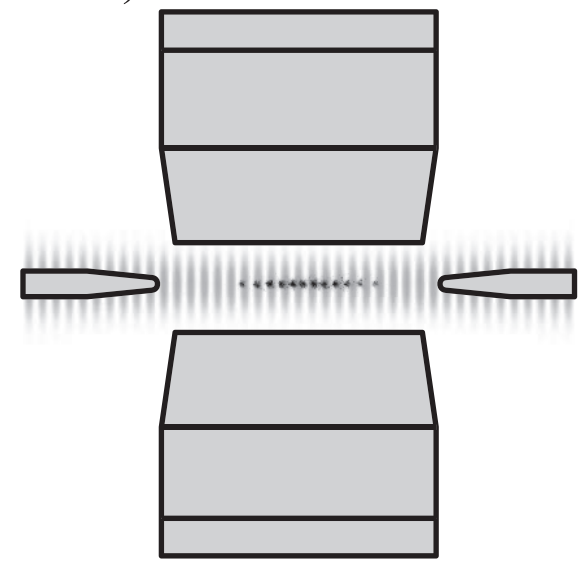

Figure 12. Schematic (not to scale) of the experimental setup suitable for simulating Frenkel Kontorova physics and observing an Aubry transition. The standing wave is applied in the axial direction, either by optical access through ring shaped endcap electrodes, or by crossing two beams at an angle, such that the counter-propagating component is aligned parallel to the weak trap axis. Part a) shows the view along the ion chain, part b) shows the setup as seen by an imaging detector used for measuring the ion fluorescence and for the determination of ion positions and formfactor

periodicity is approximately $3.1 \mu \mathrm{m}$ corresponding for example to two beams at $\lambda=1064 \mathrm{~nm}$ each enclosing an angle of $\alpha \approx 80^{\circ}$ with the weak trap axis.

The position of the lattice has to be fixed relative to the ions which means that the relative phase of the two counter-propagating running waves has to be constant. This requires stability and potentially active feedback on mirrors. To maintain a constant depth of the sinusoidal potential along the chain, the Rayleigh range or would ideally be large compared to the chain length, meaning that the waist would be large. The required strength of the standing wave $K_{c}$ to observe an Aubry transitions corresponding to a depth of $0.6 \mathrm{~K}$ ions separated by $5 \mu \mathrm{m}$ which is much deeper than in neutral atom experiments. On the other hands the trap frequencies are much higher in ion trap experiments and thus the time scales for a measurement are shorter, allowing for lasers, that are closer to the atomic resonance and in turn requiring less power. The challenging power requirements can be lessened, when the light cycles in a resonant cavity, however, with a more demanding stability.

The effective Planck constant as given in equation (55) contains the lattice constant and can in principle be changed by adjusting either the wavelength of the light that forms the sinusoidal dipole potential or the angle between the intersecting beams. To maintain the golden mean density, the trap frequency would be varied simultaneously. For parameters given above, however, the effective Planck constant is approximately $3 \cdot 10-5$ (calculated for Ca ions [García-Mata et al. 2007]) and due to the weak dependence on the lattice constant (see equation 55) ion trap experiments are likely to be restricted to the semi-classical regime with $\hbar_{\text {eff }} \ll 1$, hardly accessible for QMC calculations [García-Mata et al. 2007].

The experiment would start with an ion chain cooled to the ground state. Ramping the standing wave along the weak trap axis adiabatically up to the desired final strength ensures that the system remains in its ground state and the equilibrium positions and thus the formfactor can be measured.

Beside of the general interest in Frenkel-Kontorova physics, an axial standing wave as 
discussed above might also prove to be useful in the context of quantum information with ion traps since the strong gap in the phonon spectrum found in the pinned phase might allow for the protection of quantum gates against decoherence [García-Mata et al. 2007].

\section{Quantum Fields and relativistic effects}

\subsection{Introduction}

The way to prove a physical theory is to compare its predictions with the observations. This has been the case with the cosmological models where effects like the red shift or the homogeneous background radiation constitute observations in accordance with a description of an expanding universe and the particle creation. But to perform a truly cosmological experiment is out of the reach of our current technology, in particular one that reproduces the early stages of the universe characterized with an extremely high energy density and a rapid expansion.

In other cases like with relativistic particles, it is possible to make experiments were electrons for example are driven to almost the speed of light. But some interesting effects are still out of reach of our measurement capabilities. For example the Zitterbewegung, predicted from the Dirac equation. It states that a freely moving spin- $1 / 2$ particle, in absence of external potentials, is subject to a helicoidal motion around the main direction of propagation. Another counterintuitive effect, the Klein paradox [Klein 1929] states that an electron could transmit unimpeded through a potential barrier. But this effect has not been observed yet with elementary particles.

\subsection{Simulating the Unruh effect}

6.2.1. Methods Following the work of Unruh [Unruh 1976] it was shown that in the vacuum of a Minkowski (flat) universe an accelerated observer would measure a thermal spectrum of particles whose temperature is proportional to his acceleration. Later this effect was generalized by Gibbons and Hawking [Gibbons \& Hawking 1977] for a curved de Sitter space. The de Sitter space is a massless curved spacetime that is isotropic and homogeneous in space, and which looks like the Minkowski space locally [Bergström \& Goobar 2004]. The de Sitter space is also a cosmological model that describes an exponential expansion of the universe due to a non vanishing and positive cosmological constant $\Lambda$.

The scalar field describing a massless inflating universe with a single global mode can be imitated by the quantized motion of a single ion around its equilibrium position. The phonon excitations of the ion would simulate the field quanta of the universe. The ion itself can be used as a detector by coupling its vibrational state to an easily readable internal state.

In the case of an expanding de Sitter universe a comoving quanta field detector would experience a Doppler frequency shift of the form $\nu(t)^{\Lambda}=\nu_{0}^{\Lambda} \exp (-\sqrt{3 \Lambda} t)$ [Birrell \& Davies 1984]. The spectrum measured with this detector is equivalent to the one measured by a thermally excited inertial detector with a temperature $T=\hbar \sqrt{3 \Lambda} / k_{B}$ [Gibbons \& Hawking 1977].

In the case of a single ion trapped in a time dependent potential that varies in the form $\nu(t)=\nu_{0} \exp (-\kappa t)$, a similar effect can be measured. A pseudo-temperature of $k_{B} T=\hbar \kappa / 2 \pi$ is associated with the average occupation number of the vibrational energy levels.

The coupling between internal and vibrational states of the ion can be created by a laser field described in the dipole and rotating wave approximation by the interaction-picture 
Hamiltonian [Alsing et al. 2005, James 1998],

$$
H_{I}=\hbar \Omega k z(t)\left[\sigma^{-} e^{-i \Delta t}-\sigma^{+} e^{i \Delta t}\right] .
$$

Here, $\sigma^{+}$and $\sigma^{-}$are the raising and lowering spin-1/2 operators, $\Delta$ is the detuning of the laser relative to the atomic transition and $\Omega$ is the Rabi frequency, respectively. The laser wave vector $\vec{k}$ is pointing along the direction in which the ion's vibrational motion is excited (here the $z$-direction). We assume an anisotropic trap (e.g., a linear trap) where, by choosing the detuning $\Delta$ and the wave vector $\vec{k}$, the experimenter can control which vibrational motion to excite.

The evolution of the position operator $z(t)$ is deduced by solving the equations of motion of the ion in its harmonic trap neglecting its coupling with the light field. In the Heisenberg representation and with

$$
H=\frac{p^{2}}{2 m}+\frac{m}{2} \nu(t)^{2} z^{2},
$$

we have [Menicucci \& Milburn 2007]

$$
\begin{gathered}
\dot{z}=-\frac{i}{\hbar}[H, z]=\frac{p_{z}}{m} \\
\dot{p_{z}}=-\frac{i}{\hbar}\left[H, p_{z}\right]=m \nu(t)^{2} z
\end{gathered}
$$

After the opening of the harmonic potential $\nu(t)$ during a time $T$ in which the interaction is on, a vibrational phonon can be mapped into the excited $|1\rangle$ internal state. The probability of exciting the internal state of the ion calculated with first order perturbation theory and in the slow $\kappa \ll \nu_{0}$ and long-time $\nu_{0} e^{-\kappa T} \ll \kappa$ frequency variation for the trap potential is [Menicucci \& Milburn 2007],

$$
\begin{aligned}
P^{(1)}(\Delta) & =\frac{1}{\hbar^{2}} \int_{0}^{T} d t_{1} \int_{0}^{T} d t_{2}\left\langle 0\left|H_{I}\left(t_{1}\right)\right| 1\right\rangle\left\langle 1\left|H_{I}\left(t_{2}\right)\right| 0\right\rangle \\
& =\left(\Omega \eta_{0}\right)^{2} \frac{2 \pi \nu_{0}}{\kappa \Delta^{2}} \frac{1}{\left(e^{2 \pi \Delta / \kappa}-1\right)^{2}}
\end{aligned}
$$

When the trap is opened to simulate the expansion of the universe, the ion is used as a detector to measure the thermal spectrum from the phonon distribution. A particular case is when the laser is in resonance with the red sideband $(\Delta \approx \nu)$. The ion acts like a phonon detector that absorbs the measured particle (together with a photon) in order to produce an internal excitation of the ion. When the laser is in resonance with blue sideband $(\Delta \approx-\nu)$, the ion acts like an unconventional detector that emits a phonon to get excited. The ratio of the excitation probabilities gives the experimental signature of the ion thermal distribution [Wineland \& Itano 1979, Monroe, Meekhof, King, Jefferts, Itano, Wineland \& Gould 1995],

$$
\frac{P_{\text {red }}}{P_{\text {blue }}}=\frac{\bar{n}}{\bar{n}+1}=e^{-\hbar \nu_{0} / k_{B} T}
$$

where $\bar{n}$ is the average phonon occupation value. From the excitation probabilities of equation 60 the ratio between the red and the blue sidebands is given by [Menicucci \& Milburn 2007],

$$
\frac{P^{(1)}(\Delta)}{P^{(1)}(-\Delta)}=e^{-2 \pi \Delta / \kappa} .
$$

Therefore the temperature associated with the exponential opening of the trap potential is given by $T=\hbar \kappa / 2 \pi k_{B}$. This temperature is equivalent to the Gibbons-Hawking temperature $T=\hbar \sqrt{3 \Lambda} / k_{B}$ [Gibbons \& Hawking 1977].

The rise of the temperature of the trapped ion is due to the trap opening. At the beginning of the trap opening the evolution is adiabatic since the trap frequency obeys the condition 
$|\dot{\nu}| / \nu \ll \nu$. Indeed, the expansion constant was selected small compared to the initial trap frequency $\kappa \ll \nu_{0}$. At a later time $T$ chosen following the condition $\nu_{0} e^{-\kappa T} \ll \kappa$ the evolution turns to be non adiabatic. Indeed, the trap frequency is going to be so weak that even a small rate of change would be non-adiabatic. The ion that initially was in the ground state is excited to higher vibrational levels because of the non-adiabatic reduction of the confining potential.

6.2.2. Experiment In this case we measure the temperature associated with the ion phonon distribution. This temperature is related to the Gibbons-Hawking temperature.

To perform this experiment a single trapped ion is cooled to the vibrational ground state level. Next the ion is pumped into its electronic ground level that is related to the vacuum state of the universe. Then, the trap potential, initially at the frequency $\nu_{0}$, is exponentially reduced $\nu(t)=\nu_{0} \exp (-\kappa t)$. During the opening of the trap, Raman laser beams between hyperfine levels or a narrow frequency laser tuned to a quadrupole transition are set in resonance with the red or the blue sideband. It should be noticed that the coupling field must be in resonance with the sideband during the trap opening. After some time, the ion internal state is measured by fluorescence using the shelving technique with the help of an additional laser beam in resonance with the excited internal state.

The trap potential is then raised and the sequence can be repeated for the opposite sideband. The height of the red and blue sidebands are compared and the temperature dependence on the trap expansion coefficient $\kappa$ is measured.

A typical value of the initial trap frequency $\nu_{0} \simeq 1 \mathrm{MHz}$ can be used to perform this experiment. Therefore the expansion coefficient can be selected like $\kappa \simeq 1 \mathrm{kHz}$ and the expansion time $T$ of several ms. These are typical parameters in current ion trap experiments.

Difficulties that may arise when performing such an experiment are associated with unwanted heating of the ion caused, first, by a possible unintentional displacement of the trap minimum and by anharmonic terms in the trap potential, and second, by fluctuating patch potentials. The former could be solved by compensating the anharmonicities and the displacement of the trap potential by tuning appropriate compensating fields for every value of the time-varying trap frequency. The latter could be overcame by performing the experiment during a short enough time so that the heating can be neglected.

\subsection{Simulating the cosmological particle creation}

6.3.1. Methods The standard model describes the particle creation from the vacuum fluctuations during the early stages of the universe. Since the expansion of the universe is non adiabatic, an initial ground state will be excited evolving into a squeezed state of entangled pairs of particles. The created particle pairs are simulated by entangled phonon pairs created by the excitation of the ground ionic motional level as the trap potential is non-adiabatically varied.

One can use a real massless scalar field $\phi$ as a an approximation to describe an expanding or contracting universe. Using the Friedman-Lemaitre-Robertson-Walker metric [Birrell \& Davies 1984], $d s^{2}=a^{6}(t) d t^{2}-a^{2}(t) d \mathbf{r}^{2}$ one finds that the wave equation describing the universe reads [Schützhold et al. 2007],

$$
\left(\frac{\partial^{2}}{\partial t^{2}}+\left[a^{4}(t) \mathbf{k}^{2}+\zeta a^{6}(t) \Re(t)\right]\right) \phi_{\mathbf{k}}=0
$$

where the Ricci (curvature) scalar $\Re$ is coupled to the field via the dimensionless parameter $\zeta$. Each mode $\mathbf{k}$ represents a harmonic oscillator with a time dependent potential $a^{4}(t) \mathbf{k}^{2}+$ $\zeta a^{6}(t) \Re(t)$. 
In the case of a chain of ions trapped in a transversally strongly confining linear trap with a time dependent axial trap frequency $\nu_{z}(t)$, the position $z_{i}$ of the $i$ th ion follows the equation of motion

$$
\ddot{z}_{i}+\nu_{z}^{2}(t) z_{i}=\gamma \sum_{j \neq i} \frac{\operatorname{sign}(\mathrm{i}-\mathrm{j})}{\left(z_{i}-z_{j}\right)^{2}}
$$

This equation can be solved classically by the use of the scaling ansatz,

$$
z_{i}(t)=b(t) z_{i}^{0}
$$

where $z_{i}^{0}$ are the initial equilibrium positions. The scaling factor $b(t)$ evolves following

$$
\left(\frac{\partial^{2}}{\partial t^{2}}+\nu_{z}^{2}(t)\right) b(t)=\frac{\nu_{z}^{2}(t=0)}{b^{2}(t)}
$$

The quantum fluctuations are introduced by splitting the position operator $\hat{z}_{i}$ into its classical evolution $b(t) z_{i}^{0}$ and a quantum fluctuation term $\delta \hat{z}_{i}(t)$,

$$
\hat{z}_{i}(t)=b(t) z_{i}^{0}+\delta \hat{z}_{i}(t) .
$$

Replacing equation 67 in equation 64 and linearising later over $\delta \hat{z}_{i}(t)$ (since the displacement of the ions can be assumed small) one obtains after normal mode expansion [Schützhold et al. 2007],

$$
\left(\frac{\partial^{2}}{\partial t^{2}}+\left[\nu_{z}^{2}(t)+\frac{\nu_{\kappa}^{2}}{b^{3}(t)}\right]\right) \delta \hat{z}_{\kappa}=0
$$

for the phonon modes $\kappa$. One can recognize the similarities between equation 63 and equation 68 The scalar field $\phi_{\mathbf{k}}$ behaves in the same way as the quantum fluctuation of the ions (phonon) $\delta \hat{z}_{\kappa}$ and each mode $\mathbf{k}$ of the field has a direct analogy with the phonon frequency of the ions $\nu_{\kappa}$.

It has been shown that a non-adiabatic change in the trap potential can create squeezed states [Heinzen \& Wineland 1990].

6.3.2. Experiment The simulation can be performed in a system composed of, for example, two hyperfine states $|0\rangle$ and $|1\rangle$ of an ion. The states should be coupled by a two-photon Raman transition or by an RF field in the presence of a magnetic gradient. Notice that the pairs of Raman beams or the RF field not only couple the two hyperfine states $|0\rangle$ and $|1\rangle$ but also the vibrational levels of the ion $|n\rangle=(|n=1\rangle,|n=2\rangle, \ldots)$. For this simulation one uses three different coupling fields, one coupled to the second red sideband (a) $|0, n+2\rangle \rightarrow|1, n\rangle$, another to the first red sideband (b) $|0, n+1\rangle \rightarrow|1, n\rangle$ and the last one to the carrier transition (c) $|0, n\rangle \leftrightarrow|1, n\rangle$. An additional level $|e\rangle$ is used for fluorescence detection of the population in level $|0\rangle$. The laser pulse (d) $|0\rangle \rightarrow|e\rangle$ drives a dipole transition that does not resolve the vibrational levels as the Raman beams or RF field do.

A non-adiabatic increase of the strength of trap potential confining the ion will simulate the universe expansion. After non-adiabatically changing the trap potential, the ions motional state will be a squeezed state where only even (i.e., symmetric with respect to the trap minimum) vibrational levels contribute. Thus, the signature of the squeezed state is the population of even vibrational levels. Measuring the population of the $|n=2\rangle$ vibrational level should be sufficient, since the population in higher vibrational levels is expected to be negligible. In order to measure the population of the $n=2$ state one performs a series of pulses described in figure 13 that couple the motional state to the easily readable internal state of the ion. 


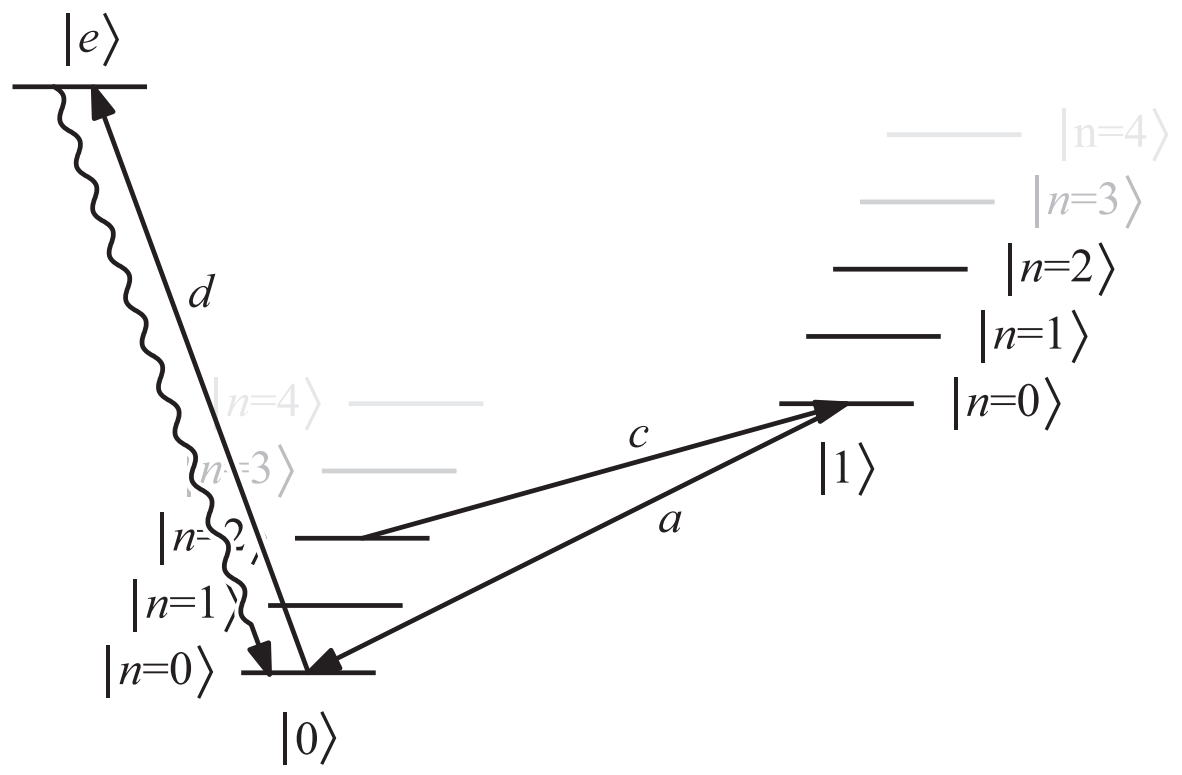

Figure 13. A possible energy level diagram and the transitions used to measure the population in the $n=2$ level. If the ion is found in level $|0, n=2\rangle$ after the trap contraction, then applying a $\pi$ second red sideband pulse (c) followed by a $\pi$ carrier pulse (a) brings the ion to the state $|0, n=2\rangle$. When the ion is in this latter level, fluorescence can be seen by using the detection laser $(d)$. On the contrary if the ion is initially in $|0, n=0\rangle$ or $|0, n=1\rangle$ the first pulse $(c)$ would not change the state of the ion since it does not have enough energy to be in resonance with the $|1\rangle$ energy level but the subsequent carrier pulse $(c)$ transfer the population to the corresponding vibrational levels of $|1\rangle$. Then no fluorescence can be seen when the detection laser $(d)$ is applied. Note that the transitions $a$ and $c$ should be narrow enough not to couple the adjacent vibrational levels and that the detection laser drives a dipole transition that couples many of the vibrational levels.

Since cooling is more efficient for strong confinement, it is advantageous to begin with a strongly confining potential and cool to the ground state of motion. Then, after an adiabatic lowering of the trap potential it is ramped back up non-adiabatically to its initial strength.

To discriminate against any classical heating, i.e. parametric or other classical perturbations, the probability of populating the $n=1$ level can be measured in a similar way as shown in figure 13 The comparison between both probabilities gives the proof that a squeezed state is generated, if a higher probability for populating the $n=2$ level than the $n=1$ is obtained.

\subsection{Simulating the Dirac Equation}

6.4.1. Methods Relativistic effects related to the solution of the Dirac equation can be simulated in trapped ions systems as proposed in [Bermudez et al. 2007] and [Lamata et al. 2007], in particular phenomena like the Zitterbewegung (helicoidal motion of the free Dirac particle as a consequence of the non-commutativity of its velocity operator components) or the Klein paradox (transmission of a relativistic particle of mass $m$ through a potential edge of height $V>2 m c^{2}$ with nearly no reflection).

In order to simulate the Dirac equation in $3+1$ dimensions for a spin- $1 / 2$ particle in a single trapped ion, L. Lamata et al. proposed to design a Hamiltonian that reproduces the same 
dynamics. For a free electron in three dimensions the Dirac formalism uses a bispinor that is a vector valued wave function of four components. In order to represent all the components one can use a four level system with states $|a\rangle,|b\rangle,|c\rangle,|d\rangle$ that correspond to four internal levels of the ion. The bispinor is defined as [Lamata et al. 2007],

$$
|\Psi\rangle:=\Psi_{a}|a\rangle+\Psi_{b}|b\rangle+\Psi_{c}|c\rangle+\Psi_{d}|d\rangle=\left(\begin{array}{c}
\Psi_{a} \\
\Psi_{b} \\
\Psi_{c} \\
\Psi_{d}
\end{array}\right)
$$

The dynamic of the system is generated by the pairwise coupling of the internal levels with the center of mass vibrational levels whose energies are determined by the trap frequencies $\nu_{x}, \nu_{y}, \nu_{z}$. Again, three common types of interaction are used. A carrier interaction coherently couples two ionic internal levels without changing the external motion of the ion for example $|a, n\rangle \leftrightarrow|c, n\rangle$. A Jaynes-Cummings (JC) and an anti-Jaynes-Cummings (AJC) interactions resonantly couple the ionic internal with the vibrational levels. The former induce the red sideband transition and the later the blue one for example $|a, n+1\rangle \leftrightarrow|c, n\rangle$ and $|a, n\rangle \leftrightarrow|c, n+1\rangle$ respectively. The corresponding interaction Hamiltonians under the rotating wave and Lamb-Dicke approximations are,

$$
\begin{aligned}
& H_{\sigma}=\hbar \Omega\left(\sigma^{+} e^{i \phi}+\sigma^{-} e^{-i \phi}\right) \\
& H_{r}=\hbar \eta \tilde{\Omega}\left(\sigma^{+} a e^{i \phi_{r}}+\sigma^{-} a^{\dagger} e^{-i \phi_{r}}\right) \\
& H_{b}=\hbar \eta \tilde{\Omega}\left(\sigma^{+} a^{\dagger} e^{i \phi_{b}}+\sigma^{-} a e^{-i \phi_{b}}\right)
\end{aligned}
$$

Here again $\sigma^{+}$and $\sigma^{-}$are the raising and lowering ionic spin-1/2 operators, $a^{\dagger}$ and $a$ are the creation and annihilation operators associated with the motional state of the ion and $\eta=\sqrt{\hbar k^{2} / 2 M \nu}$ is the Lamb-Dicke parameter. The Rabi oscillation frequencies for the blue and red sideband transitions were set to the same value $\tilde{\Omega}$ and to $\Omega$ for the carrier transition.

When only the carrier coupling is applied, with the appropriate choice of the phase $\phi$ one obtains,

$$
H_{\sigma_{j}}=\hbar \Omega_{j} \sigma_{j}
$$

with $j=\{x, y\}$ and $\sigma_{j}$ being the Pauli matrices.

When the blue and red sideband couplings are used simultaneously, the resulting Hamiltonian is the sum of $H_{r}$ and $H_{b}$ from equation 70 By choosing the phases of the sideband fields like $\phi_{b}-\phi_{r}=\pi$ one obtains,

$$
H_{\sigma_{j}}^{p}= \pm i \hbar \eta \Delta \tilde{\Omega} \sigma_{j}\left(a-a^{\dagger}\right) .
$$

Here, $\Delta=\sqrt{\hbar / 2 M \nu}$ is the spread in position of the ground wave function. The operator $\sigma_{j}$ is the Pauli matrix along the $j=\{x, y\}$ component of the spin. It is not related to the vibrational directions of motion but to the ionic internal transitions.

The selection of the Pauli matrix $\sigma_{x}=\sigma^{+}+\sigma^{-}$or $\sigma_{y}=i\left(\sigma^{-}-\sigma^{+}\right)$as well as the sign of the Hamiltonian is performed by an additional condition of the applied red and blue sidebands phases. Even if a condition on their difference was already used, one needs a supplementary condition to completely define the phases. The sum of the phases of the applied fields $\phi_{s}=\left(\phi_{b}+\phi_{r}\right) / 2$ determines the sign of the Hamiltonian, negative for $\phi_{s}=\{\pi, 3 / 2 \pi\}$ and positive for $\phi_{s}=\{0,1 / 2 \pi\}$ whereas the Pauli matrix $\sigma_{j}$ is selected along $j=x$ for $\phi_{s}=\{0, \pi\}$ and along $j=y$ for $\phi_{s}=\{\pi / 2,3 / 2 \pi\}$ (see figure 14). The phase $\phi_{s}$ itself does not modify the dynamics of the system when the previous Hamiltonian is considered alone, 
but it is essential to build the Dirac like Hamiltonian composed of many elements couplings as in equation 72 The dynamics of equation 72 are based on a spin dependent coupling between the internal and vibrational states that can also be used for the creation of entangled states of spin and motion. This coupling has already been applied in previous experiments [Haljan et al. 2005, Sackett et al. 2000].

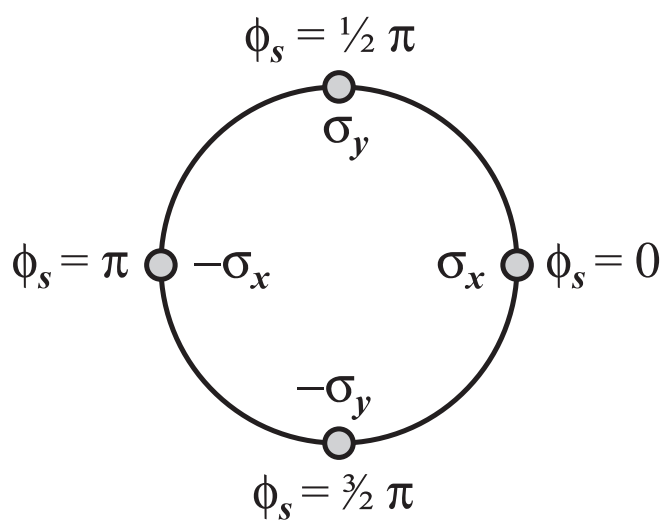

Figure 14. The selection of the phases of the red and blue sidebands $\phi_{r}$ and $\phi_{b}$ fields are important since they shape the form of Hamiltonian. In particular the sum of the phases $\phi_{s}=\left(\phi_{r}+\phi_{b}\right) / 2$ defines the direction of the spin operator and also the global sign of the Hamiltonian 72

The Hamiltonian of equation 72 constitutes the basic block to construct the Dirac Hamiltonian in $(3+1)$ dimensions. Since the spin-1/2 particle to simulate has a momentum with components in all three spatial directions, one needs to consider Hamiltonian terms along all of these directions. The operators $a^{\dagger}$ and $a$ can be associated with the three normal trap frequencies and therefore with the motion along the three trap axes. One needs to distinguish $a_{\ell}^{\dagger}$ and $a_{\ell}$ for every axis $\ell=x, y, z$. The same consideration should be made for the LambDicke parameter $\eta_{\ell}$, the spread in position of the ground state wave function $\Delta_{\ell}$ and the Rabi frequency $\Omega_{\ell}$ that also need to be defined along the direction of the applied interaction. The difference between the creation and annihilation operators can be written in terms of the momentum operator $p_{\ell}=i \hbar\left(a_{\ell}^{\dagger}-a_{\ell}\right) / \Delta_{\ell}$. Following the previous considerations, the Hamiltionian of equation 72 can be rewritten as,

$$
H_{\sigma_{j}}^{p_{\ell}}=2 \eta_{\ell} \Delta_{\ell} \tilde{\Omega}_{\ell} \sigma_{j} p_{\ell} .
$$

In order to simulate the Dirac dynamics employing four internal states of an ion, the transitions $|a\rangle \leftrightarrow|c\rangle,|a\rangle \leftrightarrow|d\rangle,|b\rangle \leftrightarrow|c\rangle$ and $|b\rangle \leftrightarrow|d\rangle$ are employed. The corresponding spin- $1 / 2$ operators of equation 73 need to be defined for the specific transition $\sigma_{j}^{a c}, \sigma_{j}^{a d}, \sigma_{j}^{b c}$ and $\sigma_{j}^{b d}$ and therefore the corresponding Hamiltonian from equation 73 can be written as $H_{\sigma_{j}(a c)}^{p_{\ell}}, H_{\sigma_{j}(a d)}^{p_{\ell}}, H_{\sigma_{j}(b c)}^{p_{\ell}}$ and $H_{\sigma_{j}(b d)}^{p_{\ell}}$. In the case of the carrier interaction of equation 71 two internal transitions are used, giving the following Hamiltonians $H_{\sigma_{\ell}(a c)}$ and $H_{\sigma_{\ell}(b d)}$.

With the correct selection of the direction and phase for the different fields, one can tailor the desired Hamiltonian as a linear combination of $H_{\sigma_{\ell}}$, and $H_{\sigma_{j}}^{p_{\ell}}$ yielding, for instance [Lamata et al. 2007],

$$
\begin{aligned}
& H_{D}=H_{\sigma_{x}(a d)}^{p_{x}}+H_{\sigma_{x}(b c)}^{p_{x}}+H_{\sigma_{y}(a d)}^{p_{y}}-H_{\sigma_{y}(b c)}^{p_{y}}+ \\
& +H_{\sigma_{x}(a c)}^{p_{z}}-H_{\sigma_{x}(b d)}^{p_{z}}+H_{\sigma_{y}(a c)}+H_{\sigma_{y}(b d)} .
\end{aligned}
$$


It should be noticed that all the Rabi oscillation frequencies for the sideband and carrier transitions $\tilde{\Omega}$ and $\Omega$, the spread in position $\Delta$ and the Lamb-Dicke parameter $\eta$ are selected equal in all the components of the previous Hamiltonian for reasons that will be explained later. This means that the fields employed to drive the various couplings should have the same relative strength for all the directions of space and the ion trap should be spherical. Therefore, one may obtain a Hamiltonian $H_{D}$ that is symmetric with respect to all directions of space. The Hamiltonian $H_{D}$ can be written in a $4 \times 4$ matrix representation in a base defined by the four internal states of the ion (see eq. 69),

$$
H_{D}=\left(\begin{array}{cc}
0 & 2 \eta \Delta \tilde{\Omega}(\vec{\sigma} \cdot \vec{p})-i \hbar \Omega \\
2 \eta \Delta \tilde{\Omega}(\vec{\sigma} \cdot \vec{p})+i \hbar \Omega & 0
\end{array}\right)
$$

where each element represents a $2 \times 2$ matrix. This Hamiltonian can be compared with the "supersymmetric" representation of the Dirac Hamiltonian [Thaller 1992],

$$
H_{D}=\left(\begin{array}{cc}
0 & c(\vec{\sigma} \cdot \vec{p})-i m c^{2} \\
c(\vec{\sigma} \cdot \vec{p})+i m c^{2} & 0
\end{array}\right) .
$$

The speed of light $c$ and the electron rest energy $m c^{2}$ are related to the coupling strengths $\Omega$ and $\tilde{\Omega}$ following,

$$
c=2 \eta \Delta \tilde{\Omega} \quad \text { and } m c^{2}=\hbar \Omega .
$$

The previous selection of symmetric couplings applied along all the directions of space represents in a simulation the isotropy of the velocity of light.

One can recognize the similarities between these two Hamiltonians, making possible the simulation of a quantum relativistic evolution of a spin-1/2 particle with an ion-trap experiment.

If the dynamics created by the Hamiltonian of equation 75 were reproduced in an experiment with a four level system using Raman beams, it would require 14 pairs of Raman lasers, two for each element of the form $H_{\sigma_{j}}^{p_{\ell}}$ (see equation 73) and one for each carrier element $H_{\sigma_{\ell}}$ (see equation 71). Of course, some simplifications can be applied, like modulating the frequency of the beams to generate the blue and the red sideband transitions with a single pair of beams. But it should be stressed the need of controlling their phases independently and the minimal requirement of having at least two pairs of beams (modulated in frequency) with a resulting k-vector along each one of the trap axes (see figure 15).

In order to render the simulation achievable with current experiments, one can simplify the $3+1$ dimensional Hamiltonian of equation 76 and work with $2+1$ dimensions [Bermudez et al. 2007], or simplify even more to $1+1$ dimensions that still reproduce the most important effects of the Dirac equation. The resulting $1+1$ dimensional Hamiltonian becomes, after a $\pi / 2$ rotation around the $x$-axis that changes $\sigma_{y}$ into $\sigma_{z}$ [Lamata et al. 2007],

$$
H_{D}^{(1)}=2 \eta \Delta \tilde{\Omega} \sigma_{x} p_{x}+\hbar \Omega \sigma_{z} .
$$

This Hamiltonian operates on the Dirac "spinor" $\left|\Psi^{(1)}\right\rangle=\Psi_{a}^{(1)}|a\rangle+\Psi_{b}^{(1)}|b\rangle$ build with a positive $\left(\Psi_{a}^{(1)}\right)$ and a negative $\left(\Psi_{b}^{(1)}\right)$ kinetic energy component [Thaller 1992]. The term $2 \eta \Delta \tilde{\Omega} \sigma_{x} p_{x}$ results from the application of blue and red sideband fields. It is just the sum of $H_{b}$ and $H_{r}$ with phases $\phi_{b}=-\pi / 2$ and $\phi_{r}=\pi / 2$. The last term $\hbar \Omega \sigma_{z}$ can be obtained, for instance, by applying an off-resonant laser pulse that induces an ac-Stark shift. Because of the reduction to one spatial dimension, the trapping potential should also be one-dimensional, that is, a linear trap with strong radial confinement compared to the axial one would be well suited.

The experimental requirements for simulating the $1+1$ Dirac Hamiltonian are substantially reduced and achievable in current experiments. 


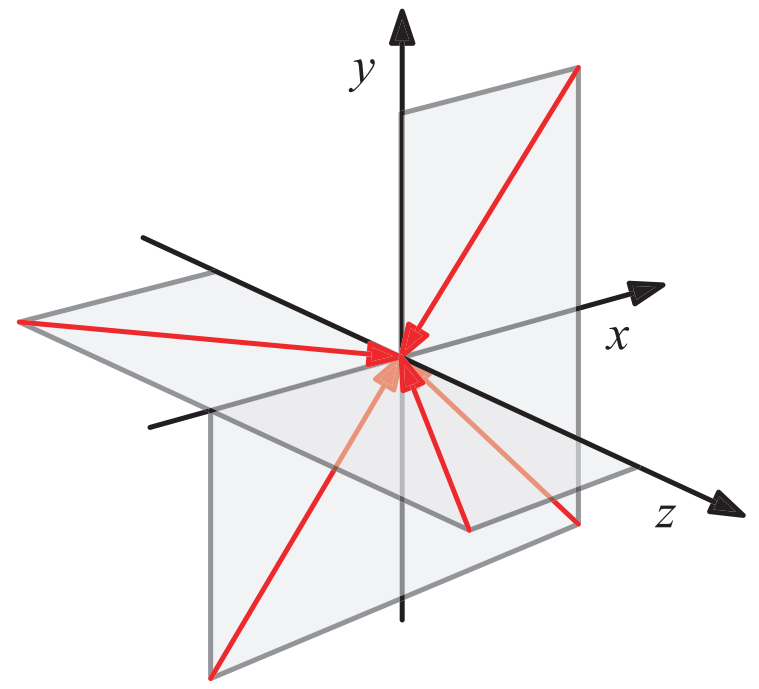

Figure 15. Possible laser configuration to build the Dirac Hamiltonian with Raman transitions. In order to build a symmetric Hamiltonian as the one of equation 75 the wave vectors must be the same along the three trap axis. For simplification of the lasers requirements only one wavelength can be used. Then the four levels to perform the simulation need to be close enough in energy i.e. two Zeeman split Hyperfine levels as proposed in [Lamata et al. 2007]. Therefore the angles subtended by every pair of Raman beams must be the same. Additionally the beams need to be modulated with the correct frequency to drive the desired transitions.

6.4.2. Experiment for the simulation of the Zitterbewegung By numerically solving the Dirac equation, it is possible to visualize peculiar behaviour of the probability density function. One of this peculiarities is the Zitterbewegung where the position of a particle oscillates even in the absence of external potentials. Usually this relativistic effect is not seen in experiments since the frequency is on the order $10^{21} \mathrm{~Hz}$ and the amplitude of order $10^{-3} \AA$ for a real particle such as an electron.

When simulating the Zitterbewegung with trapped ions the frequency and amplitude of the oscillatory motion for an ion with average momentum $p_{0}$ are given by [Lamata et al. 2007]

$$
\begin{aligned}
& \omega_{\mathrm{ZB}} \approx \sqrt{2 \eta^{2} \tilde{\Omega}^{2} p_{0}^{2} / \hbar^{2}+\Omega^{2}}, \\
& R_{\mathrm{ZB}}=\frac{\eta \hbar^{2} \tilde{\Omega} \Omega \Delta}{4 \eta^{2} \tilde{\Omega}^{2} \Delta^{2} p_{0}^{2}+\hbar^{2} \Omega^{2}} .
\end{aligned}
$$

From equation 79, one can obtain a measurable output with frequencies ranging from zero to some Megahertz and amplitudes that can go to a thousand Angstroms varying the typical parameters of Rabi frequencies and trap depths. It can be noticed that the amplitude of the Zitterwebegung can be made independent of the Rabi frequencies if $\eta \tilde{\Omega} \approx \Omega$ indicating that this effect could be seen even with small laser fields.

In order to observe the Zitterbewegung one needs to determine the momentum and the position of the ion for different times. In previous experiments [Meekhof et al. 1996a, Meekhof et al. 1996b] the state of motion of the ion was determined by measuring the population distribution of the Fock states. From the red sideband excitation probability as a function of time one can fit the different frequency components associated with the 
contribution of every vibrational level. Therefore, the determination of the distribution over Fock vibrational states requires many data points. Another possibility could be to measure the Wigner function by instantaneous measurements, even in the presence of a thermal bath, based on the proposal in [Santos et al. 2007].

To reduce the number of data points to acquire, one can just measure the expected value of the generalized quadrature $Y_{\phi}=\left(a e^{-i \phi}-a^{\dagger} e^{i \phi}\right) / 2$ as proposed by [Lougovski et al. 2006]. Indeed one does not need to measure the whole wave function to know the position or momentum of the ion, it is enough to measure the expected value. The angle $\phi$ is related to the internal spin state $\left|+_{\phi}\right\rangle=\left(|a\rangle+e^{i \phi}|b\rangle\right)$ where the ion is initialized. Additionally, a JC interaction needs to be applied (see $H_{r}$ in equation 70) which couples the vibrational state to the internal state where the measurement is performed.

The probability $P_{b}$ of finding the ion in the state $|b\rangle$ is given by (see [Bastin et al. 2006]),

$$
\begin{aligned}
\frac{d}{d t} P_{b} & =\frac{1}{i \hbar}\left\langle\left[|b\rangle\langle b|, H_{0}+H_{r}\right]\right\rangle+\left\langle\frac{\partial}{\partial t} \mid b\right\rangle\langle b \mid\rangle \\
& =\frac{1}{i \hbar}\left\langle\left[|b\rangle, H_{r}\right]\right\rangle
\end{aligned}
$$

The state of the ion at time $t=t_{0}$ is given by $\rho_{\mathrm{m}}\left(t_{0}\right) \otimes\left|+_{\phi}\right\rangle\left\langle+_{\phi}\right|$, where $\rho_{m}$ is density matrix for the vibrational levels. Then the probability of finding the ion in state $|b\rangle$ at time $t=t_{0}$ is given by,

$$
\begin{aligned}
\left.\frac{d}{d t} P_{b}\right|_{t=t_{0}} & =\left.\frac{1}{i \hbar}\left\langle\left[|b\rangle\langle b|, H_{r}\right]\right\rangle\right|_{t=t_{0}} \\
& =\operatorname{Tr}\left[\rho_{\mathrm{m}}\left(t_{0}\right) \otimes\left|+_{\phi}\right\rangle\left\langle+_{\phi}\right|\left[|b\rangle\langle b|, H_{r}\right]\right] \\
& =\eta \tilde{\Omega}\left\langle Y_{\phi}\right\rangle .
\end{aligned}
$$

Therefore the generalized quadrature can be written in dimensionless time $\tau=\eta \tilde{\Omega} t$ as,

$$
\left\langle Y_{\phi}\right\rangle=\left.\frac{d}{d \tau} P_{b}^{+_{\phi}}\right|_{\tau=0}
$$

When choosing $\phi=-\pi / 2$ and $\phi=0$ the position and momentum operators are measured, respectively. Notice that only two measurements of the probability of finding the ion in state $|b\rangle$ are needed to be taken to determine the position or momentum of the ion.

In order to perform the simulation of the Zitterbewegung the ion can be cooled to the vibrational ground level, although it is not required, since it is also possible to measure the Zitterbewegung of a Doppler cooled ion. Then the internal state of the ion is set into $|a\rangle$. The next step is to excite the ion to a given motional state, i.e. a Fock state [Meekhof et al. 1996a, Meekhof et al. 1996b], a coherent state [Carruthers \& Nieto 1965], a thermal state [Stenholm 1986], a squeezed state [Heinzen \& Wineland 1990, Cirac et al. 1993], or an arbitrary state. The relativistic-like dynamic ruled by the interaction $H_{D}$ (see equation 78) is generated by turning on the fields resonant with the blue and red sidebands at time $t=0$. The time evolution of the ion ends at $t=t_{0}$ when the interaction generated by the Hamiltonian $H_{D}$ is turned off. Simultaneously, the internal state of the ion is driven to $\left|+{ }_{\phi}\right\rangle$ by the application of an external field, then a JC coupling (see $H_{r}$ in equation 70) is applied to map the ion motional state into its internal state. At time $t=t_{0}+\tau$ with $\tau \ll 2 \pi / \omega_{Z B}$ the population in the level $|b\rangle$ is measured by detecting resonance fluorescence. The previous sequence is repeated with the same parameters for a given number of repetitions to find the probability $P_{b}^{+\phi}\left(t_{0}+\tau\right)$. Then, the probability $P_{b}^{+\phi}$ at $t=t_{0}$ is also measured (without the need of applying the JC coupling). From the two last probabilities the expectation of the generalized quadrature can be deduced. The whole sequence is then repeated for different $t$ to extract the trajectory of the ion. 
6.4.3. Experiment of the simulation of the Klein's paradox The Klein paradox [Klein 1929] is a relativistic effect described by the Dirac equation. It states that an electron can be transmitted with almost no reflection through a potential step with a height $V$ bigger than twice the electron rest energy $m c^{2}$.

In the Dirac formalism the particle kinetic energy $E$ takes into account the rest mass and therefore the electron momentum is given by

$$
p=\frac{1}{c} \sqrt{(E-V)^{2}-m^{2} c^{4}} .
$$

When $|E-V|<m c^{2}$ the momentum is imaginary and the wave function exponentially decays. However, when $V \geq E+m c^{2}$, the momentum is real and one obtains a transmitted wave. This solution lies in the negative kinetic energy range $E<-m c^{2}+V$ and therefore is associated with a electron-positron pair production. The positron sees an inverted potential step and therefore its motion is not impeded (see figure 16).

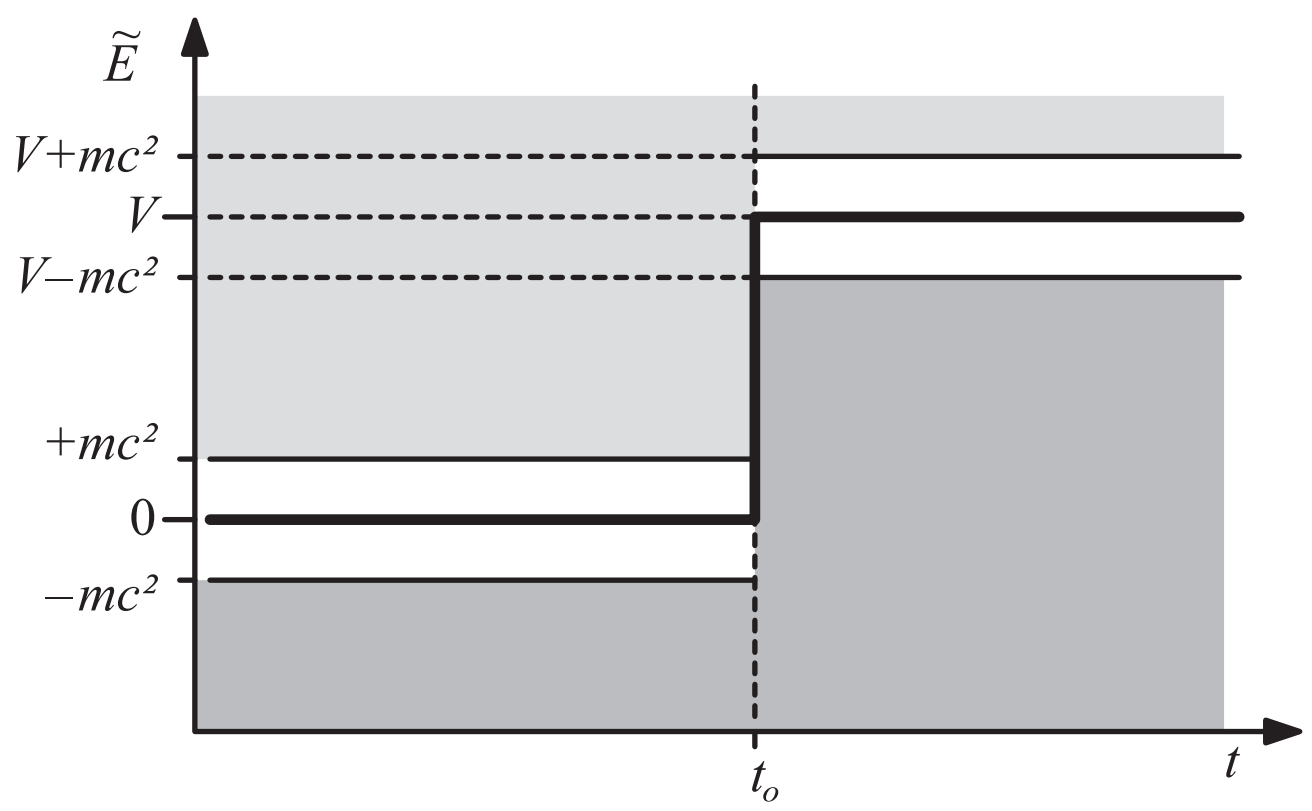

Figure 16. Potential Step (thick line). The white areas represent the forbidden regions where the solutions of the Dirac equation are exponentially decaying. The light and dark gray areas correspond to the positive and negative energy regions respectively. An electron with energy $m c^{2}<E<V-m c^{2}$ is found initially in the positive energy region. For $t>t_{0}$, after the potential step is risen, the energy lies in the negative energy region allowing the creation of a hole in the Dirac sea. Picture adapted from [Schwabl 1997].

The transmission through a potential step can be reproduced by the Hamiltonian,

$$
H_{V}^{(1)}=H_{D}^{(1)}+V(|a\rangle\langle a|+| b\rangle\langle b|) .
$$

The last term is the potential step $V(|a\rangle\langle a|+| b\rangle\langle b|)$ that is set at the time $t=t_{0}$. It can be implemented for example by the use of a detuned laser that produces the same stark shift in both levels.

The experimental protocol is summarized in the following steps. First, the ions are cooled to the ground state $|a\rangle$. Then the JC and AJC couplings, described in equation 78 , are applied at $t=0$ by turning on the red and blue sideband excitation fields. Next, the 
potential step is turned rapidly on at time $t=t_{0}$. Last, the population in the state $|b\rangle$ is measured by fluorescence. The probability of finding the ion in the state $|b\rangle$ is obtained after several repetitions and is equivalent of the transmission probability through the step potential.

\section{Acknowledgments}

We thank R. Schmied and E. Solano for stimulating discussions and comments on the manuscript. We acknowledge financial support by the European Union (IP QAP and STREP Microtrap), by the Deutsche Forschungsgemeinschaft and by secunet AG.

\section{References}

Alsing P M, Dowling J P \& Milburn G J 2005 Phys. Rev. Lett. 94(22), 220401-4.

Amico L, Fazio R, Osterloh A \& Vedral V 2008 Rev. Mod. Phys. 80(2), 517-60.

Anderson M H, Ensher J R, Matthews M R, Wieman C E \& Cornell E A 1995 Science 269(5221), 198-201.

Aubry S \& Le Daeron P Y 1983 Physica D 8, 381-422.

Bardeen J, Cooper L N \& Schrieffer J R 1957 Phys. Rev. 108(5), 1175.

Barjaktarevic J P, Milburn G J \& McKenzie R H 2005 Phys. Rev. A 71(1), 012335-5.

Barrett M D, Chiaverini J, Schätz T, Britton J, Itano W M, Jost J D, Knill E, Langer C, Leibfried D, Ozeri R \& Wineland D J 2004 Nature (London) 429(6993), 737-739.

Bastin T, von Zanthier J \& Solano E 2006 J. Phys. B 39(3), 685-693.

Bergström L \& Goobar A 2004 Cosmology and Particle Astrophysics second edition edn Praxis Publishing Ltd. Chichester.

Bermudez A, Martin-Delgado M A \& Solano E 2007 Phys. Rev. A 76(4), 041801-4.

Birkl G, Kassner S \& Walther H 1992 Nature (London) 357(6376), 310-313.

Birrell N D \& Davies P C W 1984 Quantum Fields in Curved Space Cambridge Monographs on Mathematical Physics Cambridge University Press Cambridge.

Blatt R \& Wineland D 2008 Nature (London) 453(7198), 1008-1015.

Borgonovi F, Guarneri I \& Shepelyansky D L 1989 Phys. Rev. Lett. 63(19), 2010.

Braun O M \& Kivshar Y S 1998 Phys. Rep. 306(1-2), 1-108.

Braun O M \& Kivshar Y S 2004 The Frenkel-Kontorova Model: Concepts, Methods, and Applications Springer Berlin.

Braungardt S, Sen A, Sen U \& Lewenstein M 2007 Phys. Rev. A 76(4), 042307-9.

Brüser D n.d. to be published .

Brush S G 1967 Rev. Mod. Phys. 39(4), 883.

Cai J, Popescu S \& Briegel H J 2008 arxiv:0809.4906 .

Carruthers P \& Nieto M M 1965 Am. J. Phys. 33(7), 537.

Chiaverini J \& Lybarger W E 2008 Phys. Rev. A 77(2), 11

Ciaramicoli G, Galve F, Marzoli I \& Tombesi P 2005 Phys. Rev. A 72(4), 042323.

Ciaramicoli G, Marzoli I \& Tombesi P 2007 Phys. Rev. A 75(3), 032348-10.

Ciaramicoli G, Marzoli I \& Tombesi P 2008 Phys. Rev. A 78(1), 012338.

Cirac J I, Parkins A S, Blatt R \& Zoller P 1993 Phys. Rev. Lett. 70(5), 556.

Cirac J I \& Zoller P 1995 Phys. Rev. Lett. 74(20), 4091.

Cirac J L \& Zoller P 2000 Nature (London) 404(6778), 579-581.

Clark R J, Lin T, Brown K R \& Chuang I L 2008 arXiv:0809.2824v1 [quant-ph] .

Das A \& Chakrabarti B K 2005 Quantum annealing and related optimization methods Lecture Notes in Physics Springer Berlin.

Davis K B, Mewes M O, Andrews M R, van Druten N J, Durfee D S, Kurn D M \& Ketterle W 1995 Phys. Rev. Lett. $\mathbf{7 5}(22), 3969$.

Davis K B, Mewes M O, Joffe M A, Andrews M R \& Ketterle W 1995 Phys. Rev. Lett. 74(26), 5202.

Dehmelt H 1981 in 'Thirty Fifth Annual Frequency Control Symposium. 1981' pp. 596-601.

Deng X L, Porras D \& Cirac J I 2005 Phys. Rev. A 72(6), 063407-11.

Deng X L, Porras D \& Cirac J I 2008 Phys. Rev. A 77(3), 033403-11.

Deslauriers L, Olmschenk S, Stick D, Hensinger W K, Sterk J \& Monroe C 2006 Phys. Rev. Lett. 97(10), 103007.

Diedrich F, Bergquist J C, Itano W M \& Wineland D J 1989 Phys. Rev. Lett. 62, 403-406.

Drewsen M, Brodersen C, Hornekær L, Hangst J S \& Schifffer J P 1998 Phys. Rev. Lett. 81(14), 2878.

Dubin D H E 1993 Phys. Rev. Lett. 71(17), 2753.

Feynman R 1982 Int. J. Theor. Phys. 21(6), 467-488. 
Feynman R 1986 Found. Phys. 16(6), 507.

Fisher M P A, Weichman P B, Grinstein G \& Fisher D S 1989 Phys. Rev. B 40(1), 546.

Fisher R n.d. private communication .

Fishman S, Chiara G D, Calarco T \& Morigi G 2008 Phys. Rev. B 77(6), 064111.

Frenkel Y \& Kontorova T 1938 Phys. Z. Sowietunion 13, 1.

Friedenauer A, Schmitz H, Glueckert J T, Porras D \& Schätz T 2008 Nat. Phys. 4(10), 757-761.

Gaetan A, Miroshnychenko Y, Wilk T, Chotia A, Viteau M, Comparat D, Pillet P, Browaeys A \& Grangier P 2009 Nat. Phys. 5(2), 115-118.

García-Mata I, Zhirov O V \& Shepelyansky D L 2007 Eur. Phys. J. D 41(2), 325-330.

Ghosh P K 1995 Ion Traps Oxford University Press.

Gibbons G W \& Hawking S W 1977 Phys. Rev. D 15(10), 2738-2751.

Giorgini S, Pitaevskii L P \& Stringari S 2008 Rev. Mod. Phys. 80(4), 1215.

Girardeau M 1960 J. Math. Phys. (N.Y.) 1(6), 516-523.

Gitterman M \& Halpern V 2004 Phase Transitions: A Brief Account with Modern Applications World Scientific Singapore.

Greiner M, Mandel O, Esslinger T, Hänsch T W \& Bloch I 2002 Nature (London) 415(6867), 39-44.

Grotendorst J, Marx D \& Muramatsu A 2002 Quantum Simulations of Complex Many-Body Systems: from Theory to Algorithms Forschungszentrum Jülich Jülich.

Hackermuller L, Hornberger K, Brezger B, Zeilinger A \& Arndt M 2004 Nature (London) 427(6976), 711-714.

Haljan P C, Brickman K A, Deslauriers L, Lee P J \& Monroe C 2005 Phys. Rev. Lett. 94(15), 153602.

Hebb D O 1949 The organization of behavior Wiley New York.

Heinzen D J \& Wineland D J 1990 Phys. Rev. A 42(5), 2977.

Heisenberg W 1928 Z. Phys. 49(9), 619-636.

Hensinger W K, Olmschenk S, Stick D, Hucul D, Yeo M, Acton M, Deslauriers L, Monroe C \& Rabchuk J 2006 Appl. Phys. Lett. 88(3), 034101.

Häffner H, Hänsel W, Roos C F, Benhelm J, Chek-al kar D, Chwalla M, Körber T, Rapol U D, Riebe M, Schmidt P O, Becher C, Gühne O, Dür W \& Blatt R 2005 Nature (London) 438(7068), 643-646.

Hänsch T W \& Schawlow A L 1975 Opt. Commun. 13, 68.

Hofstetter W, Cirac J I, Zoller P, Demler E \& Lukin M D 2002 Phys. Rev. Lett. 89(22), 220407.

Holland M, Kokkelmans S J J M F, Chiofalo M L \& Walser R 2001 Phys. Rev. Lett. 87(12), 120406.

Home J P \& Steane A M 2006 Quant. Inf. Comp. 6(4-5), 289-325.

Hopfield J J 1982 Proc Natl Acad Sci U S A 79(8), 2554-2558.

Hubbard J 1963 Proc. R. Soc. London, Ser. A 276(1365), 238-257.

Huber G, Deuschle T, Schnitzler W, Reichle R, Singer K \& Schmidt-Kaler F 2008 New J. Phys. 10(1), 013004.

Hucul D, Yeo M, Hensinger W K, Rabchuk J, Olmschenk S \& Monroe C 2008 Quant. Inf. Comp. 8, 0501.

Hugh D M \& Twamley J 2005 Phys. Rev. A 71(1), 012315.

Igloi F, Peschel I \& Turban L c 1993 Adv. Phys. 42(6), 683 - 740.

Ising E 1925 Z. Phys. 31(1), 253-258.

James D F V 1998 Appl. Phys. B 66(2), 181-190.

Jane E, Vidal G, Dür W, Zoller P \& Cirac J I 2003 Quant. Inf. Comp. 3.

Johanning M, Braun A, Timoney N, Elman V, Neuhauser W \& Wunderlich C 2009 Phys. Rev. Lett. 102(7), 073004.

Jonathan D \& Plenio M B 2001 Phys. Rev. Lett. 87(12), 127901.

Jonathan D, Plenio M B \& Knight P L 2000 Phys. Rev. A 62(4), 042307.

Kielpinski D, Monroe C \& Wineland D J 2002 Nature (London) 417(6890), 709-711.

Kjærgaard N \& Drewsen M 2003 Phys. Rev. Lett. 91(9), 095002.

Klein O 1929 Z. Phys. A 53(3), 157-165.

Labaziewicz J, Ge Y, Antohi P, Leibrandt D, Brown K R \& Chuang I L 2008 Phys. Rev. Lett. 100(1), 013001.

Lamata L, Leon J, Schatz T \& Solano E 2007 Phys. Rev. Lett. 98(25), 253005-4.

Leggett A J, Chakravarty S, Dorsey A T, Fisher M P A, Garg A \& Zwerger W 1987 Rev. Mod. Phys. 59(1), 1.

Leibfried D, DeMarco B, Meyer V, Lucas D, Barrett M, Britton J, Itano W M, Jelenkovic B, Langer C, Rosenband T \& Wineland D J 2003 Nature (London) 422(6930), 412-415.

Leibfried D, DeMarco B, Meyer V, Rowe M, Ben-Kish A, Britton J, Itano W M, Jelenkovic B, Langer C, Rosenband T \& Wineland D J 2002 Phys. Rev. Lett. 89(24), 247901.

Leibfried D, Knill E, Seidelin S, Britton J, Blakestad R B, Chiaverini J, Hume D B, Itano W M, Jost J D, Langer C, Ozeri R, Reichle R \& Wineland D J 2005 Nature (London) 438(7068), 639-642.

Leibfried D, Meekhof D M, King B E, Monroe C, Itano W M \& Wineland D J 1996 Phys. Rev. Lett. 77(21), 42814285.

Leibfried D, Meekhof D M, Monroe C, King B E, Itano W M \& Wineland D J 1997 Journal of Modern Optics 44(11), 2485 - 2505.

Lenz W 1920 Phys. Z. 21, 613.

Lieb E H \& Liniger W 1963 Phys. Rev. 130(4), 1605. 
Loewen M \& Wunderlich C 2004 Verhandl. DPG VI 39.

Lougovski P, Walther H \& Solano E 2006 Eur. Phys. J. D 38(3), 423-426.

Luttinger J M 1963 J. Math. Phys. (N.Y.) 4, 1154.

Meekhof D M, Monroe C, King B E, Itano W M \& Wineland D J 1996a Phys. Rev. Lett. 76(11), 1796.

Meekhof D M, Monroe C, King B E, Itano W M \& Wineland D J 1996b Phys. Rev. Lett. 77(11), 2346.

Menicucci N C \& Milburn G J 2007 Phys. Rev. A 76(5), 052105-5.

Metcalf H J \& van der Straten P 2001 Laser Cooling and Trapping Springer.

Meyrath T P, Schreck F, Hanssen J L, Chuu C S \& Raizen M G 2005 Phys. Rev. A 71(4), 041604.

Mezard M, Parisi G \& Virasoro M A 1987 Spin glass theory and beyond World Scientific Singapore.

Milburn G J 1999 arXiv:quant-ph/9908037 .

Milburn G J, Schneider S \& James D F V 2000 Fortschr. Phys. 48(9-11), 801-810.

Mintert F \& Wunderlich C 2001 Phys. Rev. Lett. 87(25), 257904.

Mintert F \& Wunderlich C 2003 Phys. Rev. Lett. 91(2), 029902.

Mitchell T B, Bollinger J J, Dubin D H, nbsp, E., Huang X P, Itano W M \& Baughman R H 1998 Science 282(5392), 1290-1293.

Müller M, Liang L, Lesanovsky I \& Zoller P 2008 New J. Phys. 10(9), 093009.

Molmer K \& Sorensen A 1999 Phys. Rev. Lett. 82, 1835-1838.

Monroe C, Meekhof D M, King B E, Itano W M \& Wineland D J 1995 Phys. Rev. Lett. 75(25), 4714.

Monroe C, Meekhof D M, King B E, Jefferts S R, Itano W M, Wineland D J \& Gould P 1995 Phys. Rev. Lett. 75(22), 4011-4014.

Monroe C, Meekhof D M, King B E \& Wineland D J 1996 Science 272(5265), 1131-1136.

Monroe C R, Meekhof D M, Leibfried D, King B E, Itano W M \& Wineland D J 1996 Optics and Photonics News 7, 13-14.

Morigi G \& Fishman S 2004a Phys. Rev. Lett. 93(17), 170602.

Morigi G \& Fishman S 2004 b Phys. Rev. E 70(6), 066141.

Morsch O \& Oberthaler M 2006 Rev. Mod. Phys. 78(1), 179.

Neuhauser W, Hohenstatt M, Toschek P E \& Dehmelt H 1980 Phys. Rev. A 22(3), 1137.

Nightingale M P \& Umrigar C J 1998 Quantum Monte Carlo Methods in Physics and Chemistry Springer.

Onsager L 1944 Phys. Rev. 65(3-4), 117.

Ospelkaus C, Langer C E, Amini J M, Brown K R, Leibfried D \& Wineland D J 2008 Phys. Rev. Lett. 101(9), 090502.

Osterloh A, Amico L, Falci G \& Fazio R 2002 Nature (London) 416(6881), 608-610.

Paredes B, Widera A, Murg V, Mandel O, Fölling S, Cirac I, Shlyapnikov G V, Hänsch T W \& Bloch I 2004 Nature (London) 429(6989), 277-281.

Peitgen H O, Jürgens H \& Saupe D 2004 Chaos and Fractals: New Frontiers of Science 2nd edn Springer New York. Pfeuty P 1970 Annals of Physics 57(1), 79-90.

Platini T, Karevski D \& Turban L 2007 J. Phys. A 40(7), 1467-1479.

Plenio M B \& Virmani S 2007 Quant. Inf. Comp. 7, 1.

Plenio M \& Huelga S 2008 New J. Phys. 10(11), 113019.

Pons M, Ahufinger V, Wunderlich C, Sanpera A, Braungardt S, Sen A, Sen U \& Lewenstein M 2007 Phys. Rev. Lett. 98(2), 023003.

Porras D \& Cirac J I 2004a Phys. Rev. Lett. 92(20), 207901.

Porras D \& Cirac J I 2004b Phys. Rev. Lett. 93(26), 263602.

Porras D, Marquardt F, Delft J v \& Cirac J I 2008 Phys. Rev. A 78(1), 010101.

Powell H F, Segal D M \& Thompson R C 2002 Phys. Rev. Lett. 89(9), 093003.

Raizen M G, Gilligan J M, Bergquist J C, Itano W M \& Wineland D J 1992 Phys. Rev. A 45(9), 6493.

Reichle R, Leibfried D, Blakestad R B, Britton J, Jost J D, Knill E, Langer C, Ozeri R, Seidelin S \& Wineland D J 2006 Fortschr. Phys. 54(8-10), 666-685.

Retzker A, Thompson R C, Segal D M \& Plenio M B 2008 Phys. Rev. Lett. 101(26), 260504.

Riebe M, Haffner H, Roos C F, Hansel W, Benhelm J, Lancaster G P T, Korber T W, Becher C, Schmidt-Kaler F, James D F V \& Blatt R 2004 Nature (London) 429(6993), 734-737.

Rojas R 1996 Neural Networks - A Systematic Introduction Springer-Verlag Berlin, New York.

Rosenband T, Hume D B, Schmidt P O, Chou C W, Brusch A, Lorini L, Oskay W H, Drullinger R E, Fortier T M, Stalnaker J E, Diddams S A, Swann W C, Newbury N R, Itano W M, Wineland D J \& Bergquist J C 2008 Science 319(5871), 1808-1812.

Rowe M A, Ben-Kish A, Demarco B, Leibfried D, Meyer V, Beall J, Britton J, Hughes J, Itano W M, Jelenkovic B, Langer C, Rosenband T \& Wineland D J 2002 Quant. Inf. Comp. 2(4), 257-271.

Sachdev S 1999 Quantum Phase Transitions Cambridge University Press Cambridge, UK.

Sackett C A, Kielpinski D, King B E, Langer C, Meyer V, Myatt C J, Rowe M, Turchette Q A, Itano W M, Wineland D J \& Monroe I C 2000 Nature (London) 404(6775), 256-259.

Santos M F, Giedke G \& Solano E 2007 Phys. Rev. Lett. 98(2), -.

Sasura M \& Steane A M 2003 Phys. Rev. A 67(6), 19. 
Schiffer J P 1993 Phys. Rev. Lett. 70(6), 818.

Schmidt-Kaler F, Häffner H, Riebe M, Gulde S, Lancaster G P T, Deuschle T, Becher C, Roos C F, Eschner J \& Blatt R 2003 Nature (London) 422(6930), 408-411.

Schmied R, Roscilde T, Murg V, Porras D \& Cirac J I 2008 New J. Phys. 10(4), 045017.

Schollwöck U, Richter J, Farnell D \& Bishop R 2004 Quantum Magnetism Lecture notes in physics 645 Springer Berlin.

Schrödinger E 1935 Naturwissenschaften 23(48), 807-812.

Schützhold R, Uhlmann M, Petersen L, Schmitz H, Friedenauer A \& Schätz T 2007 Phys. Rev. Lett. 99(20), 201301-

Schulte T, Drenkelforth S, Kruse J, Ertmer W, Arlt J J, Kantian A, Sanchez-Palencia L, Santos L, Sanpera A, Sacha K, Zoller P, Lewenstein M \& Zakrzewski J 2006 Acta Physica Polonica A 109, 89-99.

Schulz S, Poschinger U, Singer K \& Schmidt-Kaler F 2006 Fortschr. Phys. 54(8-10), 648-665.

Schwabl F 1997 Quantenmechanik für Fortgeschrittene Springer-Verlag Berlin.

Seidelin S, Chiaverini J, Reichle R, Bollinger J J, Leibfried D, Britton J, Wesenberg J H, Blakestad R B, Epstein R J, Hume D B, Itano W M, Jost J D, Langer C, Ozeri R, Shiga N \& Wineland D J 2006 Phys. Rev. Lett. 96(25), 253003-4.

Sørensen A \& Mølmer K 2000 Phys. Rev. A 62(2), 022311.

Steane A 1997 Appl. Phys. B 64(6), 623-643.

Stenholm S 1986 Rev. Mod. Phys. 58(3), 699.

Stick D, Hensinger W K, Olmschenk S, Madsen M J, Schwab K \& Monroe C 2006 Nat. Phys. 2(1), 36-39.

Thaller B 1992 The Dirac Equation Texts and Monographs in Physics Springer-Verlag Berlin.

Tolra B L, Oâ€тMHara K M, Huckans J H, Phillips W D, Rolston S L \& Porto J V 2004 Phys. Rev. Lett. 92(19), 190401.

Turchette Q A, Kielpinski D, King B E, Leibfried D, Meekhof D M, Myatt C J, Rowe M A, Sackett C A, Wood C S, Itano W M, Monroe C \& Wineland D J 2000 Phys. Rev. A 61(6), 8.

Unruh W G 1976 Phys. Rev. D 14(4), 870-892.

Urban E, Johnson T A, Henage T, Isenhower L, Yavuz D D, Walker T G \& Saffman M 2009 Nat. Phys. 5(2), 110-114.

Voit J 1995 Rep. Prog. Phys. 58, 977.

Waki I, Kassner S, Birkl G \& Walther H 1992 Phys. Rev. Lett. 68(13), 2007.

Wallentowitz S, Vogel W, Siemers I \& Toschek P E 1996 Phys. Rev. A 54(1), 943.

Wang S X, Labaziewicz J, Ge Y, Shewmon R \& Chuang I L 2008 arXiv:0811.2422v1 .

Weiss U 1999 Quantum dissipative systems World Scientific Singapore.

White S R 1993 Phys. Rev. B 48(14), 10345.

Wineland D J \& Dehmelt H 1975 Bull. Am. Phys. Soc. 20, 637.

Wineland D J \& Itano W M 1979 Phys. Rev. A 20(4), 1521-1540.

Wineland D J, Monroe C, Itano W M, King B E, Leibfried D, Myatt C \& Wood C 1998 Phys. Scr. p. 147.

Wineland D J, Monroe C, Itano W M, Leibfried D, King B E \& Meekhof D M 1998 J. Res. Natl. Inst. Stand. Technol. 103(3), 259-328.

Wunderlich C 2002 in 'Laser Physics at the Limit', H Figger, D Meschede \& C Zimmermann, eds, Springer Berlin pp. 261-271.

Wunderlich C \& Balzer C 2003 in Advances in Atomic Molecular and Optical Physics 49 Academic Press Inc San Diego pp. 293-372.

Wunderlich C, Morigi G \& Reiß D 2005 Phys. Rev. A 72(2), 023421.

Wunderlich H \& Wunderlich C 2009 arXiv:0901.0881 .

Zhirov O V, Casati G \& Shepelyansky D L 2002 Phys. Rev. E 65(2), 026220. 